Bull. Austral. Math. Soc.

$81 \mathrm{Q} 60,81 \mathrm{R} 50,81 \mathrm{U} 40,82 \mathrm{~B} 23$

VOL. 64 (2001) [445-467]

\title{
INTEGRABLE EXTENDED HUBBARD MODELS WITH BOUNDARY KONDO IMPURITIES
}

\author{
Anthony J. Bracken, Xiang-Yu Ge, Mark D. Gould \\ AND HUAN-QIANG ZHOU
}

\begin{abstract}
Three kinds of integrable Kondo impurity additions to one-dimensional $q$-deformed extended Hubbard models are.studied by means of the boundary $\mathbf{Z}_{2}$-graded quantum inverse scattering method. The boundary $K$ matrices depending on the local magnetic moments of the impurities are presented as nontrivial realisations of the reflection equation algebras in an impurity Hilbert space. The models are solved by using the algebraic Bethe ansatz method, and the Bethe ansatz equations are obtained.
\end{abstract}

\section{INTRODUCTION}

A major motivation for the study of strongly correlated electron models comes from the discovery of high- $T_{c}$ superconductivity. The Hubbard model and the $t-J$ model, which have attracted a great deal of attention in the last decade, were initiated by Anderson [1] and Zhang and Rice [20], in the context of high- $T_{c}$ superconductivity. In one dimension, Shastry [15, 16] and Essler and Korepin [3] established the integrability of the Hubbard model and $t-J$ model respectively in the framework of the Quantum Inverse Scattering Method [18]. Since then, many new exactly solvable strongly correlated electron models of interest have been proposed, such as the supersymmetric Essler-Korepin-Schoutens extended Hubbard model $[5,6]$. The latter is a $g l(2 \mid 2)$ supersymmetric generalisation of the supersymmetric $t-J$ model and can be interpreted as the Hubbard model plus moderate nearest-neighbour interactions. The complete solution of the one-dimensional super symmetric Essler-Korepin-Schoutens extended Hubbard model by the algebraic Bethe ansatz has been extensively investigated in [7] and it exhibits the so-called off-diagonal long-range order, which is relevant to superconductivity. Specifically, the mathematical issue of the completeness of the solution has been settled [14], and the physics content of the solution, low lying excitations in particular, has been studied [4].

On the other hand, the study of Kondo impurities in correlated electron hosts has received much attention recently. In $[21,23]$, we have successfully constructed integrable

Received 2nd April, 2001

This work was supported by the Australian Research Council.

Copyright Clearance Centre, Inc. Serial-fee code: 0004-9727/01 \$A2.00+0.00. 
Kondo impurity additions to three differend kinds of the extended Hubbard models in the framework of the graded boundary Quantum Inverse Scattering Method [17, 2]. This is a generalisation of our previous work on integrable Kondo impurity additions to the $t-J$ models [24, 22], which itself is an algebraic reformulation of the coordinate Bethe ansatz results $[19,12]$ (see also the work of Frahm and Slavnov [9]). More recently, boundary impurities for the generalised ( $q$-deformed) $t-J$ model have also been studied by the graded boundar Quantum Inverse Scattering Method $[\mathbf{8}, 11]$.

The purpose of the present work is to extend the investigation of [11] to the cases of the $q$-deformed extended Hubbard models. That is, integrable Kondo impurity additions with spin- $1 / 2$ coupled with three different type of $q$-deformed extended Hubbard open chains, are constructed. This is achieved by searching for operator-valued boundary $K$ matrices as nontrivial realisations of the $\mathbf{Z}_{2}$-graded reflection equation algebras in a finite dimensional quantum space which is interpretated as an impurity Hilbert space. It should be emphasised that our new non-c-number boundary $K$ matrices are highly nontrivial, in the sense that they can not be factorised into the product of a c-number boundary $K$ matrix and the corresponding local monodromy matrices. This fact characterises a difference between our construction and that of Zvyagin and Schlottamnn [13, 25]. The models we present are solved by means of the algebraic Bethe ansatz method and the Bethe ansatz equations are derived.

\section{Boundary Hamiltonian with Kondo Impurities}

Let $c_{j, \sigma}^{\dagger}$ and $c_{j, \sigma}$ denote creation and annihilation operators for conduction electrons with spin $\sigma$ at site $j$, satisfying the anti-commutation relations given by $\left\{c_{i, \sigma}^{\dagger}, c_{j, \sigma^{\prime}}\right\}=$ $\delta_{i j} \delta_{\sigma \sigma^{\prime}}$, where $i, j=1,2, \cdots, L$ and $\sigma, \sigma^{\prime}=\uparrow$, $\downarrow$. At a given lattice site $j$ there are four possible electronic states:

$$
|0\rangle, \quad|\uparrow\rangle_{j}=c_{j, \dagger}^{\dagger}|0\rangle, \quad|\downarrow\rangle_{j}=c_{j, \downarrow}^{\dagger}|0\rangle, \quad|\uparrow, \downarrow\rangle_{j}=c_{j, \downarrow}^{\dagger} c_{j, \uparrow}^{\dagger}|0\rangle .
$$

We consider the following type of Hamiltonian, describing two magnetic impurities coupled to the $q$-deformed extended Hubbard chain of Essler, Korepin and Schoutens $[5,6]$ :

$$
\begin{aligned}
H= & -\sum_{j=1}^{L-1} \sum_{\sigma}\left(c_{j, \sigma}^{\dagger} c_{j+1, \sigma}+H . c .\right)\left(1-n_{j,-\sigma}-n_{j+1,-\sigma}\right) \\
& -\sum_{j=1}^{L-1}\left(c_{j, \uparrow}^{\dagger} c_{j, \downarrow}^{\dagger} c_{j+1, \downarrow} c_{j+1, \uparrow}-S_{j}^{+} S_{j+1}^{-}+H . c .\right) \\
& +q^{-1} \sum_{j=1}^{L-1}\left(n_{j}-n_{j, \uparrow} n_{j, \downarrow}+n_{j, \downarrow} n_{j+1, \uparrow}\right)+q \sum_{j=1}^{L-1}\left(n_{j+1}-n_{j+1, \uparrow} n_{j+1, \downarrow}+n_{j, \uparrow} n_{j+1, \downarrow}\right) \\
& +2\left(q+q^{-1}\right) \sum_{j=1}^{L-1}\left(S_{j}^{z} S_{j+1}^{z}-\frac{1}{4} n_{j} n_{j+1}\right)
\end{aligned}
$$




$$
\begin{aligned}
& +\left(q-q^{-1}\right) \sum_{j=1}^{L-1}\left(n_{j, \uparrow} n_{j, \downarrow} n_{j+1, \uparrow}-n_{j, \uparrow} n_{j+1, \uparrow} n_{j+1, \downarrow}\right) \\
& +J_{a}\left(\sigma_{a}^{-} S_{1}^{+}+\sigma_{a}^{+} S_{1}^{-}\right)+V_{a} n_{1 \uparrow}+U_{a} n_{1 \downarrow}+W_{a} n_{1 \downarrow} n_{1 \uparrow} \\
& +J_{b}\left(\sigma_{b}^{-} S_{L}^{+}+\sigma_{b}^{+} S_{L}^{-}\right)+V_{b} n_{L \uparrow}+U_{b} n_{L \downarrow}+W_{b} n_{L \downarrow} n_{L \uparrow} .
\end{aligned}
$$

In the above, $S_{j}^{+}, S_{j}^{-}, S_{j}^{z}$ as usual is the vector spin operator for the conduction electrons at site $j$ satisfying the $s u(2)$ algebra and expressed as $S_{j}^{+}=c_{j, \uparrow}^{\dagger} c_{j, \downarrow}, S_{j}^{-}=c_{j, \downarrow}^{\dagger} c_{j, \uparrow}$, $S_{j}^{z}=1 / 2\left(n_{j, \uparrow}-n_{j, \downarrow}\right) ; \sigma_{g}^{ \pm}=\sigma_{g}^{x} \pm i \sigma_{g}^{y}, \sigma_{g}^{z}(g=a, b)$ are the local moments with spin- $1 / 2$ located at the left and right ends of the system respectively. Now the Kondo coupling constants $J_{g}, V_{g}, U_{g}$ and $W_{g}(g=a, b)$ should be written in terms of the arbitrary parameters $c_{9}$ :

$$
\begin{aligned}
J_{g} & =\frac{q^{c_{g}}\left(q^{2}-1\right)^{2}}{\left(q^{c_{a}}-q^{2}\right)\left(q^{c_{a}+2}-1\right)} \\
V_{g} & =\frac{q^{c_{g}+1}\left(q^{2}-1\right)\left(2 q^{c_{g}}-q^{2}-1-\left(q^{2}-1\right) \sigma_{g}^{z}\right)}{2\left(q^{c_{a}}-q^{2}\right)\left(q^{c_{a}+2}-1\right)}, \\
U_{g} & =\frac{q^{c_{g}-1}\left(q^{2}-1\right)\left(2 q^{c_{g}+2}-2 q^{4}+q^{2}-1+\left(q^{2}-1\right) \sigma_{g}^{z}\right)}{2\left(q^{c_{a}}-q^{2}\right)\left(q^{c_{a}+2}-1\right)} \\
W_{g} & =-\frac{q^{c_{g}-1}\left(q^{2}-1\right)\left(4 q^{c_{g}+2}-3 q^{4}-1-\left(q^{2}-1\right)^{2} \sigma_{g}^{z}\right)}{2\left(q^{c_{a}}-q^{2}\right)\left(q^{c_{a}+2}-1\right)}:
\end{aligned}
$$

It has been shown [10] that the bulk Hamiltonian acquires an underlying supersymmetry algebra $U_{q}(g l(2 \mid 2))$ in the minimal representation. Furthermore, open chain integrability with appropriate boundary conditions was established using the boundary Quantum Inverse Scattering Method. Let us recall that the local Hamiltonian of the supersymmetric $q$-deformed extended Hubbard model is derived from an $R$-matrix, satisfying the Yang-Baxter equation, which has the form [10]

(3) $R(u)=$

$$
\left(\begin{array}{cccccccccccccccc}
a(u) & 0 & 0 & 0 & 0 & 0 & 0 & 0 & 0 & 0 & 0 & 0 & 0 & 0 & 0 & 0 \\
0 & b(u) & 0 & 0 & c(u) & 0 & 0 & 0 & 0 & 0 & 0 & 0 & 0 & 0 & 0 & 0 \\
0 & 0 & b(u) & 0 & 0 & 0 & 0 & 0 & c(u) & 0 & 0 & 0 & 0 & 0 & 0 & 0 \\
0 & 0 & 0 & b(u) & 0 & 0 & 0 & 0 & 0 & 0 & 0 & 0 & c(u) & 0 & 0 & 0 \\
0 & d(u) & 0 & 0 & b(u) & 0 & 0 & 0 & 0 & 0 & 0 & 0 & 0 & 0 & 0 & 0 \\
0 & 0 & 0 & 0 & 0 & a(u) & 0 & 0 & 0 & 0 & 0 & 0 & 0 & 0 & 0 & 0 \\
0 & 0 & 0 & 0 & 0 & 0 & b(u) & 0 & 0 & c(u) & 0 & 0 & 0 & 0 & 0 & 0 \\
0 & 0 & 0 & 0 & 0 & 0 & 0 & b(u) & 0 & 0 & 0 & 0 & 0 & c(u) & 0 & 0 \\
0 & 0 & d(u) & 0 & 0 & 0 & 0 & 0 & b(u) & 0 & 0 & 0 & 0 & 0 & 0 & 0 \\
0 & 0 & 0 & 0 & 0 & 0 & d(u) & 0 & 0 & b(u) & 0 & 0 & 0 & 0 & 0 & 0 \\
0 & 0 & 0 & 0 & 0 & 0 & 0 & 0 & 0 & 0 & e(u) & 0 & 0 & 0 & 0 & 0 \\
0 & 0 & 0 & 0 & 0 & 0 & 0 & 0 & 0 & 0 & 0 & b(u) & 0 & 0 & -c(u) & 0 \\
0 & 0 & 0 & d(u) & 0 & 0 & 0 & 0 & 0 & 0 & 0 & 0 & b(u) & 0 & 0 & 0 \\
0 & 0 & 0 & 0 & 0 & 0 & 0 & d(u) & 0 & 0 & 0 & 0 & 0 & b(u) & 0 & 0 \\
0 & 0 & 0 & 0 & 0 & 0 & 0 & 0 & 0 & 0 & 0 & -d(u) & 0 & 0 & b(u) & 0 \\
0 & 0 & 0 & 0 & 0 & 0 & 0 & 0 & 0 & 0 & 0 & 0 & 0 & 0 & 0 & e(u)
\end{array}\right)
$$




$$
a(u)=1, \quad b(u)=\frac{q\left(q^{u}-1\right)}{q^{u}-q^{2}}, \quad c(u)=\frac{1-q^{2}}{q^{u}-q^{2}}, \quad d(u)=\frac{q^{u}\left(1-q^{2}\right)}{q^{u}-q^{2}}, \quad e(u)=\frac{q^{u+2}-1}{q^{u}-q^{2}},
$$

where $u$ is the spectral parameter.

We remark that the four possible electronic states of the vector irrep of the quantum superalgebra $U_{q}(g l(2 \mid 2))$ symmetry, are identified with

$$
|1\rangle=|0\rangle, \quad|2\rangle=c_{j, \downarrow}^{\dagger} c_{j, \uparrow}^{\dagger}|0\rangle, \quad|3\rangle=c_{j, \uparrow}^{\dagger}|0\rangle, \quad|4\rangle=c_{j, \downarrow}^{\dagger}|0\rangle
$$

and we choose to adopt the bosonic, bosonic, fermionic and fermionic grading: $[|1\rangle]=$ $[|2\rangle]=0,[|3\rangle]=[|4\rangle]=1$ on the indices labelling the basis vectors.

A second choice of integrable coupling is given by

$$
\begin{aligned}
H= & -\sum_{j=1}^{L-1} \sum_{\sigma}\left(c_{j, \sigma}^{\dagger} c_{j+1, \sigma}+H . c .\right)\left(1-n_{j,-\sigma}-n_{j+1,-\sigma}\right) \\
& -\sum_{j=1}^{L-1}\left(c_{j, \uparrow}^{\dagger} c_{j, \downarrow}^{\dagger} c_{j+1, \downarrow} c_{j+1, \uparrow}+S_{j}^{+} S_{j+1}^{-}+H . c .\right) \\
& +q \sum_{j=1}^{L-1}\left(n_{j+1}-n_{j+1, \uparrow} n_{j+1, \downarrow}\right)+q^{-1} \sum_{j=1}^{L-1}\left(n_{j}-n_{j, \uparrow} n_{j, \downarrow}\right) \\
& -\left(q+q^{-1}\right) \sum_{j=1}^{L-1}\left(S_{j}^{z} S_{j+1}^{z}+\frac{3}{4} n_{j} n_{j+1}\right)+\frac{q-q^{-1}}{2} \sum_{j=1}^{L-1}\left(n_{j, \uparrow} n_{j+1, \downarrow}-n_{j, \downarrow} n_{j+1, \uparrow}\right) \\
& -\left(q+q^{-1}\right) \sum_{j=1}^{L-1} n_{j, \uparrow} n_{j, \downarrow}\left(n_{j+1, \uparrow} n_{j+1, \downarrow}-n_{j+1}\right) \\
& -\left(q+q^{-1}\right) \sum_{j=1}^{L-1} n_{j+1, \uparrow} n_{j+1, \downarrow}\left(n_{j, \uparrow} n_{j, \downarrow}-n_{j}\right) \\
& +J_{a}\left(\sigma_{a}^{-} S_{1}^{+}+\sigma_{a}^{+} S_{1}^{-}\right)+V_{a} n_{1 \uparrow}+U_{a} n_{1 \downarrow}+W_{a} n_{1 \downarrow} n_{1 \uparrow} \\
& +J_{b}\left(\sigma_{b}^{-} S_{L}^{+}+\sigma_{b}^{+} S_{L}^{-}\right)+V_{b} n_{L \uparrow}+U_{b} n_{L \downarrow}+W_{b} n_{L \downarrow} n_{L \uparrow},
\end{aligned}
$$

In this case the dependence of the Kondo coupling constants $J_{g}, V_{g}, U_{9}$ and $W_{g}(g=a, b)$ on arbitrary parameters $c_{g}$ takes the form

$$
\begin{aligned}
J_{g} & =-\frac{q^{c_{g}}\left(q^{2}-1\right)^{2}}{\left(q^{c_{a}}-q^{2}\right)\left(q^{c_{a}+2}-1\right)} \\
V_{g} & =\frac{q^{c_{g}-1}\left(q^{2}-1\right)\left(2 q^{2-c_{g}}-q^{2}-1+\left(q^{2}-1\right) \sigma_{g}^{z}\right)}{2\left(q^{c_{a}}-q^{2}\right)\left(q^{c_{a}+2}-1\right)} \\
U_{g} & =-\frac{q^{c_{g}-1}\left(q^{2}-1\right)\left(2 q^{2-c_{g}}+q^{4}+q^{2}-2+q^{2}\left(q^{2}-1\right) \sigma_{g}^{z}\right)}{2\left(q^{c_{a}}-q^{2}\right)\left(q^{c_{a}+2}-1\right)} \\
W_{g} & =-\frac{q^{c_{g}-1}\left(q^{2}-1\right)^{3}\left(1-\sigma_{g}^{z}\right)}{2\left(q^{c_{a}}-q^{2}\right)\left(q^{c_{a}+2}-1\right)}
\end{aligned}
$$


The Hamiltonian in (5) arises from use of an $R$-matrix obtained by imposing $\mathbf{Z}_{2}$ grading on the one-dimensional $q$-deformed $U_{q}(s u(4)) R$-matrix, which reads

(7) $R(u)=$

$$
\left(\begin{array}{cccccccccccccccc}
a(u) & 0 & 0 & 0 & 0 & 0 & 0 & 0 & 0 & 0 & 0 & 0 & 0 & 0 & 0 & 0 \\
0 & b(u) & 0 & 0 & c(u) & 0 & 0 & 0 & 0 & 0 & 0 & 0 & 0 & 0 & 0 & 0 \\
0 & 0 & b(u) & 0 & 0 & 0 & 0 & 0 & c(u) & 0 & 0 & 0 & 0 & 0 & 0 & 0 \\
0 & 0 & 0 & b(u) & 0 & 0 & 0 & 0 & 0 & 0 & 0 & 0 & c(u) & 0 & 0 & 0 \\
0 & d(u) & 0 & 0 & b(u) & 0 & 0 & 0 & 0 & 0 & 0 & 0 & 0 & 0 & 0 & 0 \\
0 & 0 & 0 & 0 & 0 & a(u) & 0 & 0 & 0 & 0 & 0 & 0 & 0 & 0 & 0 & 0 \\
0 & 0 & 0 & 0 & 0 & 0 & b(u) & 0 & 0 & c(u) & 0 & 0 & 0 & 0 & 0 & 0 \\
0 & 0 & 0 & 0 & 0 & 0 & 0 & b(u) & 0 & 0 & 0 & 0 & 0 & c(u) & 0 & 0 \\
0 & 0 & d(u) & 0 & 0 & 0 & 0 & 0 & b(u) & 0 & 0 & 0 & 0 & 0 & 0 & 0 \\
0 & 0 & 0 & 0 & 0 & 0 & d(u) & 0 & 0 & b(u) & 0 & 0 & 0 & 0 & 0 & 0 \\
0 & 0 & 0 & 0 & 0 & 0 & 0 & 0 & 0 & 0 & -a(u) & 0 & 0 & 0 & 0 & 0 \\
0 & 0 & 0 & 0 & 0 & 0 & 0 & 0 & 0 & 0 & 0 & -b(u) & 0 & 0 & -c(u) & 0 \\
0 & 0 & 0 & d(u) & 0 & 0 & 0 & 0 & 0 & 0 & 0 & 0 & b(u) & 0 & 0 & 0 \\
0 & 0 & 0 & 0 & 0 & 0 & 0 & d(u) & 0 & 0 & 0 & 0 & 0 & b(u) & 0 & 0 \\
0 & 0 & 0 & 0 & 0 & 0 & 0 & 0 & 0 & 0 & 0 & -d(u) & 0 & 0 & -b(u) & 0 \\
0 & 0 & 0 & 0 & 0 & 0 & 0 & 0 & 0 & 0 & 0 & 0 & 0 & 0 & 0 & -a(u)
\end{array}\right),
$$

where $a(u), b(u), c(u), d(u)$ are the same as in (3) and $u$ is the spectral parameter. We adopt the same choice for the $\mathbf{Z}_{2}$-grading of the basis states as before.

The third choice of integrable couplings is given by

$$
\begin{aligned}
H= & -\sum_{j=1}^{L-1} \sum_{\sigma}\left(c_{j, \sigma}^{\dagger} c_{j+1, \sigma}+H . c .\right)\left(1-n_{j,-\sigma}-n_{j+1,-\sigma}\right) \\
& -\sum_{j=1}^{L-1}\left(c_{j, \uparrow}^{\dagger} c_{j, \downarrow}^{\dagger} c_{j+1, \downarrow} c_{j+1, \uparrow}+S_{j}^{+} S_{j+1}^{-}+H . c .\right) \\
& -q^{-1} \sum_{j=1}^{L-1}\left(n_{j+1}-n_{j+1, \uparrow} n_{j+1, \downarrow}+n_{j, \uparrow} n_{j+1, \downarrow}\right)-q \sum_{j=1}^{L-1}\left(n_{j}-n_{j, \uparrow} n_{j, \downarrow}+n_{j+1, \uparrow} n_{j, \downarrow}\right) \\
& -2\left(q+q^{-1}\right) \sum_{j=1}^{L-1}\left(S_{j}^{z} S_{j+1}^{z}-\frac{1}{4} n_{j} n_{j+1}\right)-\left(q+q^{-1}\right) \sum_{j=1}^{L-1} n_{j, \uparrow} n_{j, \downarrow} n_{j+1, \uparrow} n_{j+1, \downarrow} \\
& +\left(q-q^{-1}\right) \sum_{j=1}^{L-1}\left(n_{j, \uparrow} n_{j, \downarrow} n_{j+1, \uparrow}-n_{j, \uparrow} n_{j+1, \uparrow} n_{j+1, \downarrow}\right) \\
& +J_{a}\left(\sigma_{a}^{-} S_{1}^{+}+\sigma_{a}^{+} S_{1}^{-}\right)+V_{a} n_{1 \uparrow}+U_{a} n_{1 \downarrow}+W_{a} n_{1 \downarrow} n_{1 \uparrow} \\
& +J_{b}\left(\sigma_{b}^{-} S_{L}^{+}+\sigma_{b}^{+} S_{L}^{-}\right)+V_{b} n_{L \uparrow}+U_{b} n_{L \downarrow}+W_{b} n_{L \downarrow} n_{L \uparrow}
\end{aligned}
$$

where the dependence of the Kondo coupling constants $J_{g}, V_{g}, U_{g}$ and $W_{g}(g=a, b)$ on arbitrary parameters $c_{g}$ takes the form (6).

The Hamiltonian in (8) arises from use of an $R$-matrix obtained by imposing $\mathbf{Z}_{2}$ grading on the one-dimensional $q$-deformed $U_{q}(g l(3 \mid 1)) R$-matrix, which reads, 
(9) $R(u)=$

$$
\left(\begin{array}{cccccccccccccccc}
-e(u) & 0 & 0 & 0 & 0 & 0 & 0 & 0 & 0 & 0 & 0 & 0 & 0 & 0 & 0 & 0 \\
0 & b(u) & 0 & 0 & c(u) & 0 & 0 & 0 & 0 & 0 & 0 & 0 & 0 & 0 & 0 & 0 \\
0 & 0 & b(u) & 0 & 0 & 0 & 0 & 0 & c(u) & 0 & 0 & 0 & 0 & 0 & 0 & 0 \\
0 & 0 & 0 & b(u) & 0 & 0 & 0 & 0 & 0 & 0 & 0 & 0 & c(u) & 0 & 0 & 0 \\
0 & d(u) & 0 & 0 & b(u) & 0 & 0 & 0 & 0 & 0 & 0 & 0 & 0 & 0 & 0 & 0 \\
0 & 0 & 0 & 0 & 0 & a(u) & 0 & 0 & 0 & 0 & 0 & 0 & 0 & 0 & 0 & 0 \\
0 & 0 & 0 & 0 & 0 & 0 & b(u) & 0 & 0 & c(u) & 0 & 0 & 0 & 0 & 0 & 0 \\
0 & 0 & 0 & 0 & 0 & 0 & 0 & b(u) & 0 & 0 & 0 & 0 & 0 & c(u) & 0 & 0 \\
0 & 0 & d(u) & 0 & 0 & 0 & 0 & 0 & b(u) & 0 & 0 & 0 & 0 & 0 & 0 & 0 \\
0 & 0 & 0 & 0 & 0 & 0 & d(u) & 0 & 0 & b(u) & 0 & 0 & 0 & 0 & 0 & 0 \\
0 & 0 & 0 & 0 & 0 & 0 & 0 & 0 & 0 & 0 & -a(u) & 0 & 0 & 0 & 0 & 0 \\
0 & 0 & 0 & 0 & 0 & 0 & 0 & 0 & 0 & 0 & 0 & -b(u) & 0 & 0 & -c(u) & 0 \\
0 & 0 & 0 & d(u) & 0 & 0 & 0 & 0 & 0 & 0 & 0 & 0 & b(u) & 0 & 0 & 0 \\
0 & 0 & 0 & 0 & 0 & 0 & 0 & d(u) & 0 & 0 & 0 & 0 & 0 & b(u) & 0 & 0 \\
0 & 0 & 0 & 0 & 0 & 0 & 0 & 0 & 0 & 0 & 0 & -d(u) & 0 & 0 & -b(u) & 0 \\
0 & 0 & 0 & 0 & 0 & 0 & 0 & 0 & 0 & 0 & 0 & 0 & 0 & 0 & 0 & -a(u)
\end{array}\right)
$$

where $a(u), b(u), c(u), d(u), e(u)$ are the same as in (3) and $u$ is the spectral parameter. We adopt the same choice for the $\mathbf{Z}_{2}$-grading of the basis states as before.

\section{Graded Reflection Equations and Boundary $K$-Matrices}

We begin by reviewing the $\mathbf{Z}_{2}$-graded boundary Quantum Inverse Scattering Method as formulated in [2]. It should be noted that the supertrace is carried out for the auxiliary superspace $V$. The elements of the supermatrix $T(u)$ are the generators of an associative superalgebra $\mathcal{A}$ defined by the relations

$$
R_{12}(u-v) \stackrel{1}{T}(u) \stackrel{2}{T}(v)=\stackrel{2}{T}(v) \stackrel{1}{T}(u) R_{12}(u-v)
$$

where $\stackrel{1}{X} \equiv X \otimes 1, \stackrel{2}{X} \equiv 1 \otimes X$ for any supermatrix $X \in E n d(V)$. For later use, we list some useful properties enjoyed by the R-matrix:

(i) Unitarity: $R_{12}(u) R_{21}(-u)=\rho(u)$

(ii) Crossing-unitarity: $R_{12}^{s t_{2}}(-u+1) R_{21}^{s t_{2}}(u)=\tilde{\rho}(u)$

with $\rho(u), \tilde{\rho}(u)$ being some scalar functions.

In order to describe integrable models on open chains, we introduce two associative superalgebras $\mathcal{T}_{-}$and $\mathcal{T}_{+}$defined by the $R$-matrix $R\left(u_{1}-u_{2}\right)$ and the relations

$$
\begin{aligned}
& R_{12}(u-v) \mathcal{T}_{-}^{1}(u) R_{21}(u+v) \stackrel{2}{\mathcal{T}}_{-}(v) \\
&=\stackrel{2}{\mathcal{T}}_{-}(v) R_{12}(u+v) \stackrel{1}{\mathcal{T}}_{-}(u) R_{21}(u-v) \\
& R_{21}^{s t_{1} i s t_{2}}(-u+v) \stackrel{1}{\mathcal{T}}_{+}^{s t_{1}}(u)\left\{\left[R_{21}^{s t_{1}}(u+v)\right]^{-1}\right\}^{i s t_{2}} \stackrel{2}{\mathcal{T}}_{+}^{i s t_{2}}(v) \\
&=\stackrel{\mathcal{T}}{+}_{+}^{i s t_{2}}(v)\left\{\left[R_{12}^{i s t_{2}}(u+v)\right]^{-1}\right\}^{s t_{1}} \stackrel{1}{\mathcal{T}}_{+}^{s t_{1}}(u) R_{12}^{s t_{1} i s t_{2}}(-u+v)
\end{aligned}
$$


respectively. (For the background to these equations see [2].) Here the supertransposition $s t_{\alpha}(\alpha=1,2)$ is only carried out in the $\alpha$-th factor superspace of $V \otimes V$, whereas ist denotes the inverse operation of $s t_{\alpha}$. By modifying Sklyanin's arguments [2], one may show that the quantities $\tau(u)$ given by $\tau(u)=\operatorname{str}\left(\mathcal{T}_{+}(u) \mathcal{T}_{-}(u)\right)$ constitute a commutative family, that is, $[\tau(u), \tau(v)]=0$.

One can obtain a class of realisations of the superalgebras $\mathcal{T}_{+}$and $\mathcal{T}_{-}$by choosing $\mathcal{T}_{ \pm}(u)$ to be of the form

$$
\mathcal{T}_{-}(u)=T_{-}(u) \tilde{\mathcal{T}}_{-}(u) T_{-}^{-1}(-u), \quad \mathcal{T}_{+}^{s t}(u)=T_{+}^{s t}(u) \tilde{\mathcal{T}}_{+}^{s t}(u)\left(T_{+}^{-1}(-u)\right)^{s t}
$$

with

(14) $T_{-}(u)=R_{0 M}(u) \cdots R_{01}(u), \quad T_{+}(u)=R_{0 L}(u) \cdots R_{0, M+1}(u), \quad \tilde{\mathcal{T}}_{ \pm}(u)=K_{ \pm}(u)$,

where 0 labels an auxiliary superspace $V_{0}=C^{2,2}, M$ is any index between 1 and $L$, and $K_{ \pm}(u)$, called boundary $K$-matrices, are representations of $\mathcal{T}_{ \pm}$in a representation superspace $V=C^{2,2}$.

We now solve (11) and (12) for $K_{+}(u)$ and $K_{-}(u)$. For the quantum $R$-matrix (3), one can check that the boundary $K$-matrix $K_{-}(u)$ given by

$$
K_{-}(u)=\left(\begin{array}{cccc}
1 & 0 & 0 & 0 \\
0 & 1 & 0 & 0 \\
0 & 0 & A_{-}(u) & B_{-}(u) \\
0 & 0 & C_{-}(u) & D_{-}(u)
\end{array}\right),
$$

with

$$
\begin{aligned}
& A_{-}(u)=\frac{q^{2 u+4}+q^{2 u+2}-2 q^{u+c_{a}+2}-2 q^{u-c_{a}+2}+q^{4}-q^{2}+2-q^{2}\left(q^{2}-1\right)\left(q^{2 u}-1\right) \sigma_{a}^{z}}{2\left(q^{-u+c_{a}+2}-1\right)\left(q^{u-c_{a}+2}-1\right)}, \\
& B_{-}(u)=\frac{q\left(q^{2}-1\right)\left(q^{2 u}-1\right) \sigma_{a}^{-}}{\left(q^{-u+c_{a}+2}-1\right)\left(q^{u-c_{a}+2}-1\right)} \\
& C_{-}(u)=\frac{q\left(q^{2}-1\right)\left(q^{2 u}-1\right) \sigma_{a}^{+}}{\left(q^{-u+c_{a}+2}-1\right)\left(q^{u-c_{a}+2}-1\right)} \\
& D_{-}(u)=\frac{2 q^{2 u+4}-q^{2 u+2}+q^{2 u}-2 q^{u+c_{a}+2}-2 q^{u-c_{a}+2}+q^{2}+1+\left(q^{2}-1\right)\left(q^{2 u}-1\right) \sigma_{a}^{z}}{2\left(q^{-u+c_{a}+2}-1\right)\left(q^{u-c_{a}+2}-1\right)},
\end{aligned}
$$

satisfies equation (11) (see Appendix). We can check also that the boundary $K$-matrix $K_{+}(u)$,

$$
K_{+}(u)=\left(\begin{array}{cccc}
1 & 0 & 0 & 0 \\
0 & q^{2} & 0 & 0 \\
0 & 0 & A_{+}(u) & B_{+}(u) \\
0 & 0 & C_{+}(u) & D_{+}(u)
\end{array}\right),
$$


with

$$
\begin{aligned}
& A_{+}(u)=-\frac{q^{u+c_{b}+2}\left(q^{2 u+2}-2 q^{u-c_{b}+4}-2 q^{u+c_{b}}+q^{2 u}+2 q^{4}-q^{2}+1-\left(q^{2 u}-1\right)\left(q^{2}-1\right) \sigma_{b}^{z}\right)}{2\left(q^{u+4}-q^{c_{b}}\right)\left(q^{u}-q^{c_{b}}\right)}, \\
& B_{+}(u)=-\frac{q^{u+c_{b}+2}\left(q^{2 u}-1\right)\left(q^{2}-1\right) \sigma_{b}^{-}}{\left(q^{u+4}-q^{c_{b}}\right)\left(q^{u}-q^{c_{b}}\right)} \\
& C_{+}(u)=-\frac{q^{u+c_{b}+2}\left(q^{2 u}-1\right)\left(q^{2}-1\right) \sigma_{b}^{+}}{\left(q^{u+4}-q^{c_{b}}\right)\left(q^{u}-q^{c_{b}}\right)} \\
& D_{+}(u) \\
& \quad=-\frac{q^{u+c_{b}+2}\left(q^{2 u+2}-q^{2 u}+2 q^{2 u-2}-2 q^{u-c_{b}+2}-2 q^{u+c_{b}-2}+q^{2}+1+\left(q^{2 u+2}-1\right)\left(q^{2}-1\right) \sigma_{b}^{z}\right)}{2\left(q^{u+4}-q^{c_{b}}\right)\left(q^{u}-q^{c_{b}}\right)},
\end{aligned}
$$

is a solution of the equation (12).

By modifying Sklyanin's arguments [2], we can show that the boundary transfer matrix $\tau(u)$ given by

$$
\tau(u)=\operatorname{str}\left(K_{+}(u) T(u) K_{-}(u) T^{-1}(-u)\right)
$$

satisfies $\left[\tau\left(u_{1}\right), \tau\left(u_{2}\right)\right]=0$. It can be shown that the Hamiltonian (2) is related to the second derivative of the corresponding boundary transfer matrix $\tau(u)$ in (17) with respect to the spectral parameter $u$, at $u=0$ :

$$
\begin{aligned}
H=\sum_{j=1}^{L-1} H_{j, j+1}+\frac{1}{2} \stackrel{1}{K}{ }_{-}^{\prime}(0) & +\frac{1}{2(V+2 W)}\left[s t r_{0}\left(\stackrel{0}{K}+(0) G_{L 0}\right)\right. \\
& \left.+2 s t r_{0}\left(\stackrel{0}{K}_{+}^{\prime}(0) H_{L 0}\right)+s t r_{0}\left(\stackrel{0}{K}+(0)\left(H_{L 0}\right)^{2}\right)\right]
\end{aligned}
$$

with

$$
\begin{gathered}
H_{i, j}=\left.\frac{d}{d u} \check{R}_{i j}(u)\right|_{u=0}=P_{i, j} R_{i, j}^{\prime}(0), \\
V=\operatorname{str}_{0} \stackrel{0}{K}_{+}^{\prime}(0), \quad W=\operatorname{str}_{0}\left(\stackrel{0}{K}_{+}(0) H_{L 0}\right), \quad G_{i, j}=P_{i, j} R_{i, j}{ }^{\prime \prime}(0),
\end{gathered}
$$

where $P$ denotes the form of the $\mathbf{Z}_{2}$-graded permutation operator and the subscript 0 denotes the 4-dimensional auxiliary superspace $V_{0}=C^{2,2}$ with the grading $P[i]=0$ if $i=$ 1,2 and 1 if $i=3,4$.

For the second choice, we also solve (11) and (12). For the quantum $R$-matrix (7), one may check that the boundary $K$-matrix $K_{-}(u)$ given by

$$
K_{-}(u)=\left(\begin{array}{cccc}
1 & 0 & 0 & 0 \\
0 & 1 & 0 & 0 \\
0 & 0 & A_{-}(u) & B_{-}(u) \\
0 & 0 & C_{-}(u) & D_{-}(u)
\end{array}\right)
$$


with

$$
\begin{aligned}
& A_{-}(u)=\frac{q^{2 u+2}+q^{2 u}-2 q^{u+c_{a}+2}-2 q^{u-c_{a}+2}+2 q^{4}-q^{2}+1+\left(q^{2}-1\right)\left(q^{2 u}-1\right) \sigma_{a}^{z}}{2\left(q^{-u-c_{a}+2}-1\right)\left(q^{u+c_{a}+2}-1\right)}, \\
& B_{-}(u)=-\frac{q\left(q^{2}-1\right)\left(q^{2 u}-1\right) \sigma_{a}^{-}}{\left(q^{-u-c_{a}+2}-1\right)\left(q^{u+c_{a}+2}-1\right)} \\
& C_{-}(u)=-\frac{q\left(q^{2}-1\right)\left(q^{2 u}-1\right) \sigma_{a}^{+}}{\left(q^{-u-c_{a}+2}-1\right)\left(q^{u+c_{a}+2}-1\right)} \\
& D_{-}(u)=\frac{q^{2 u+4}-q^{2 u+2}+2 q^{2 u}-2 q^{u+c_{a}+2}-2 q^{u-c_{a}+2}++q^{4}+q^{2}-q^{2}\left(q^{2}-1\right)\left(q^{2 u}-1\right) \sigma_{a}^{z}}{\left(q^{-u-c_{a}+2}-1\right)\left(q^{u+c_{a}+2}-1\right)} .
\end{aligned}
$$

satisfies the graded reflection equation (11). Then the boundary $K$-matrix $K_{+}(u)$ defined by

$$
K_{+}(u)=\left(\begin{array}{cccc}
1 & 0 & 0 & 0 \\
0 & q^{2} & 0 & 0 \\
0 & 0 & A_{+}(u) & B_{+}(u) \\
0 & 0 & C_{+}(u) & D_{+}(u)
\end{array}\right)
$$

with

$$
\begin{aligned}
& A_{+}(u)=\frac{q^{u+c_{b}-2}\left(q^{2 u+2}-2 q^{u-c_{b}+2}-2 q^{u+c_{b}+6}+q^{2 u}+2 q^{6}+q^{10}-q^{8}-\left(q^{2 u}-q^{8}\right)\left(q^{2}-1\right) \sigma_{b}^{z}\right)}{2\left(q^{u+c_{b}}-1\right)\left(q^{u+c_{b}}-q^{4}\right)}, \\
& B_{+}(u)=\frac{q^{u+c_{b}-2}\left(q^{2 u}-q^{8}\right)\left(q^{2}-1\right) \sigma_{b}^{-}}{2\left(q^{u+c_{b}}-1\right)\left(q^{u+c_{b}}-q^{4}\right)} \\
& C_{+}(u)=\frac{q^{u+c_{b}-2}\left(q^{2 u}-q^{8}\right)\left(q^{2}-1\right) \sigma_{b}^{+}}{2\left(q^{u+c_{b}}-1\right)\left(q^{u+c_{b}}-q^{4}\right)} \\
& D_{+}(u)=\frac{q^{u+c_{b}-2}\left(2 q^{2 u+4}-q^{2 u+2}-q^{2 u}-2 q^{u-c_{b}+4}-2 q^{u+c_{b}+8}+q^{10}+q^{8}+\left(q^{2 u}-q^{8}\right)\left(q^{2}-1\right) \sigma_{b}^{z}\right)}{2\left(q^{u+c_{b}}-1\right)\left(q^{u+c_{b}}-q^{4}\right)}
\end{aligned}
$$

is a solution of the equation (12).

Following Sklyanin's approach [17], one defines the boundary transfer matrix (17), and then it can be shown that $\left[\tau\left(u_{1}\right), \tau\left(u_{2}\right)\right]$ is satisfied. Because $\operatorname{str}_{0} \stackrel{0}{K}+(0) \neq 0$, it can be shown that the Hamiltonian (5) can be embedded into the boundary transfer matrix $\tau(u)$ by

$$
H=\sum_{j=1}^{L-1} H_{j, j+1}+\frac{1}{2} \stackrel{1}{K}_{-}^{\prime}(0)+\frac{s t r_{0}\left(\stackrel{0}{K}+(0) H_{L 0}\right)}{s t r_{0} \stackrel{0}{K}_{+}(0)}
$$

with

$$
H_{i, j}=\left.\frac{d}{d u} P_{i, j} R_{i, j}(u)\right|_{u=0}=P_{i, j} R_{i, j}{ }^{\prime}(0),
$$

where $P$ is the $\mathbf{Z}_{2}$-graded permutation operator.

For the third choice, we also solve (11) and (12). For the quantum $R$-matrix (9), one may check that the boundary $K$-matrix $K_{-}(u)$ given by (19), satisfies the graded 
reflection equation (11). For this case the boundary $K$-matrix $K_{+}(u)$ defined by

$$
K_{+}(u)=\left(\begin{array}{cccc}
1 & 0 & 0 & 0 \\
0 & -1 & 0 & 0 \\
0 & 0 & A_{+}(u) & B_{+}(u) \\
0 & 0 & C_{+}(u) & D_{+}(u)
\end{array}\right)
$$

with

$$
\begin{aligned}
& A_{+}(u)=-\frac{q^{u+c_{b}}\left(q^{2 u+2}-2 q^{u-c_{b}+2}-2 q^{u+c_{b}+2}+q^{2 u}+2 q^{2}+q^{6}-q^{4}-\left(q^{2 u}-q^{4}\right)\left(q^{2}-1\right) \sigma_{b}^{z}\right)}{2\left(q^{u+c_{b}}-1\right)\left(q^{u+c_{b}}-q^{4}\right)}, \\
& B_{+}(u)=-\frac{q^{u+c_{b}}\left(q^{2 u}-q^{4}\right)\left(q^{2}-1\right) \sigma_{b}^{-}}{2\left(q^{u+c_{b}}-1\right)\left(q^{u+c_{b}}-q^{4}\right)}, \\
& C_{+}(u)=-\frac{q^{u+c_{b}}\left(q^{2 u}-q^{4}\right)\left(q^{2}-1\right) \sigma_{b}^{+}}{2\left(q^{u+c_{b}}-1\right)\left(q^{u+c_{b}}-q^{4}\right)} \\
& D_{+}(u)=-\frac{q^{u+c_{b}}\left(2 q^{2 u+4}-q^{2 u+2}+q^{2 u}-2 q^{u-c_{b}+4}-2 q^{u+c_{b}+4}+q^{6}+q^{4}+\left(q^{2 u}-q^{4}\right)\left(q^{2}-1\right) \sigma_{b}^{z}\right)}{2\left(q^{u+c_{b}}-1\right)\left(q^{u+c_{b}}-q^{4}\right)},
\end{aligned}
$$

is a solution of the equation (12).

It can be shown for this case also that the Hamiltonian (8) can be embedded into the boundary transfer matrix $\tau(u)$ as in (21).

\section{The Bethe Ansatz Equations}

Having established the quantum integrability of the models, let us first diagonalise the Hamiltonian (2) by means of the algebraic Bethe ansatz method [17, 22]. We introduce the 'doubled' monodromy matrix

$$
\mathcal{T}_{-}(u)=T(u) K_{-}(u) T^{-1}(-u) \equiv\left(\begin{array}{cccc}
\mathcal{A}(u) & \mathcal{B}_{1}(u) & \mathcal{B}_{2}(u) & \mathcal{B}_{3}(u) \\
\mathcal{C}_{1}(u) & \mathcal{D}_{11}(u) & \mathcal{D}_{12}(u) & \mathcal{D}_{13}(u) \\
\mathcal{C}_{2}(u) & \mathcal{D}_{21}(u) & \mathcal{D}_{22}(u) & \mathcal{D}_{23}(u) \\
\mathcal{C}_{3}(u) & \mathcal{D}_{31}(u) & \mathcal{D}_{32}(u) & \mathcal{D}_{33}(u)
\end{array}\right)
$$

Substituting this $\mathcal{T}(u)$ into the reflection equation (11), we may derive the following commutation relations,

$$
\begin{aligned}
\check{\mathcal{D}}_{b d}\left(u_{1}\right) \mathcal{B}_{c}\left(u_{2}\right)= & \frac{\left(q^{u_{1}-u_{2}}-q^{2}\right)\left(q^{u_{1}+u_{2}}-q^{4}\right)}{q^{2}\left(q^{u_{1}-u_{2}}-1\right)\left(q^{u_{1}+u_{2}}-q^{2}\right)} r\left(u_{1}+u_{2}-2\right)_{g h}^{e b} r\left(u_{1}-u_{2}\right)_{c d}^{i h} \mathcal{B}_{e}\left(u_{2}\right) \check{\mathcal{D}}_{g i}\left(u_{1}\right) \\
& +\frac{q^{u_{1}+u_{2}}\left(1-q^{2}\right)\left(q^{2 u_{1}}-q^{4}\right)\left(q^{2 u_{2}}-1\right)}{\left(q^{u_{1}+u_{2}}-q^{2}\right)\left(q^{2 u_{1}}-q^{2}\right)\left(q^{2 u_{2}}-q^{2}\right)} r\left(2 u_{1}-2\right)_{c d}^{g b} \mathcal{B}_{g}\left(u_{1}\right) \mathcal{A}\left(u_{2}\right) \\
& -\frac{q^{u_{1}-u_{2}}\left(1-q^{2}\right)\left(q^{2 u_{1}}-q^{4}\right)}{q^{2}\left(q^{u_{1}-u_{2}}-1\right)\left(q^{2 u_{1}}-q^{2}\right)} r\left(2 u_{1}-2\right)_{i d}^{g b} \mathcal{B}_{g}\left(u_{1}\right) \check{\mathcal{D}}_{i c}\left(u_{2}\right), \\
(24) & \\
\mathcal{A}\left(u_{1}\right) \mathcal{B}_{b}\left(u_{2}\right)= & \frac{\left(q^{u_{1}-u_{2}+2}-1\right)\left(q^{u_{1}+u_{2}}-1\right)}{\left(q^{u_{1}-u_{2}}-1\right)\left(q^{u_{1}+u_{2}}-q^{2}\right)} \mathcal{B}_{b}\left(u_{2}\right) \mathcal{A}\left(u_{1}\right) \\
& -\frac{\left(1-q^{2}\right)\left(q^{2 u_{2}}-1\right)}{\left.(25) \quad \mathcal{B}^{2 u_{2}}-q^{2}\right)\left(q^{u_{1}-u_{2}}-1\right)}\left(u_{1}\right) \mathcal{A}\left(u_{2}\right)-\frac{1-q^{2}}{q^{u_{1}+u_{2}}-q^{2}} \mathcal{B}_{d}\left(u_{1}\right) \check{\mathcal{D}}_{d b}\left(u_{2}\right) .
\end{aligned}
$$


Here $\mathcal{D}_{b d}(u)=\check{\mathcal{D}}_{b d}(u)+\left(q^{2 u}\left(1-q^{2}\right) /\left(q^{2 u}-q^{2}\right)\right) \delta_{b d} \mathcal{A}(u)$ and the matrix $r(u)$, which in turn satisfies the quantum Yang-Baxter equation, takes the form,

$$
\begin{aligned}
& r_{11}^{11}(u)=1, \quad r_{22}^{22}(u)=r_{33}^{33}(u)=\frac{q^{u+2}-1}{q^{u}-q^{2}} \\
& r_{12}^{12}(u)=r_{13}^{13}(u)=r_{21}^{21}(u)=r_{23}^{23}(u)=r_{31}^{31}(u)=r_{32}^{32}(u)=\frac{q\left(q^{u}-1\right)}{q^{u}-q^{2}} \\
& r_{21}^{12}(u)=r_{31}^{13}(u)=-r_{32}^{23}(u)=\frac{1-q^{2}}{q^{u}-q^{2}} \\
& r_{12}^{21}(u)=r_{13}^{31}(u)=-r_{23}^{32}(u)=\frac{q^{u}\left(1-q^{2}\right)}{q^{u}-q^{2}}
\end{aligned}
$$

Choose the Bethe state $|\Omega\rangle$ as

$$
|\Omega\rangle=\mathcal{C}_{i_{1}}\left(u_{1}\right) \cdots \mathcal{C}_{i_{N}}\left(u_{N}\right)|0\rangle F^{i_{1} \cdots i_{N}}
$$

with $|0\rangle$ being the pseudovacuum, where the indices $i_{j}$ run over the values $1,2,3$ and $F^{i_{1} \cdots i_{N}}$ is a function of the spectral parameters $u_{j}$. Applying the transfer matrix $\tau(u)$ to the state $|\Omega\rangle$, we have $\tau(u)|\Omega\rangle=\Lambda(u)|\Omega\rangle$, with the eigenvalue,

$$
\begin{aligned}
& \Lambda(u)=\frac{q^{2}\left(q^{2 u}-1\right)\left(q^{u+2}-q^{c_{b}}\right)\left(q^{u}-q^{c_{b}+2}\right)}{\left(q^{2 u}-q^{2}\right)\left(q^{u}-q^{c_{b}}\right)\left(q^{u+4}-q^{c_{b}}\right)} \prod_{j=1}^{N} \frac{\left(q^{u+u_{j}}-1\right)\left(q^{u-u_{j}+2}-1\right)}{q^{2}\left(q^{u+u_{j}-2}-1\right)\left(q^{u-u_{j}}-1\right)} \\
& \quad+\frac{q^{2}\left(q^{2 u}-1\right)}{q^{2 u}-q^{2}}\left(\frac{q\left(q^{u}-1\right)}{q^{u}-q^{2}}\right)^{2 L} \prod_{j=1}^{N} \frac{\left(q^{u+u_{j}-2}-q^{2}\right)\left(q^{u-u_{j}}-q^{2}\right)}{q^{2}\left(q^{u+u_{j}-2}-1\right)\left(q^{u-u_{j}}-1\right)} \Lambda^{(1)}\left(u ;\left\{u_{j}\right\}\right),
\end{aligned}
$$

provided the parameters $\left\{u_{j}\right\}$ satisfy

$$
\begin{aligned}
& \frac{\left(q^{2 u_{j}}-1\right)\left(q^{u_{j}+2}-q^{c_{b}}\right)\left(q^{u_{j}}-q^{c_{b}+2}\right)}{\left(q^{2 u_{j}-2}-q^{2}\right)\left(q^{u_{j}}-q^{c_{b}}\right)\left(q^{u_{j}+4}-q^{c_{b}}\right)}\left(\frac{q^{u_{j}}-q^{2}}{q\left(q^{u_{j}}-1\right)}\right)^{2 L} \\
& =\prod_{\substack{j=1 \\
i \neq j}}^{N} \frac{\left(q^{u_{j}+u_{i}-2}-q^{2}\right)\left(q^{u_{j}-u_{i}}-q^{2}\right)}{\left(q^{u_{j}+u_{i}}-1\right)\left(q^{u_{j}-u_{i}+2}-1\right)} \Lambda^{(1)}\left(u_{j} ;\left\{u_{i}\right\}\right) .
\end{aligned}
$$

Here $\Lambda^{(1)}\left(u ;\left\{u_{j}\right\}\right)$ is the eigenvalue of the nested boundary transfer matrix $\tau^{(1)}(u)$

$$
\tau^{(1)}(u)=\operatorname{str}\left(K_{+}^{(1)}(u) T^{(1)}\left(u,\left\{u_{j}\right\}\right) K_{-}^{(1)}(u) T^{(1)^{-1}}\left(-u,\left\{u_{j}\right\}\right)\right)
$$

which arises out of the $r$ matrices from the first term in the right hand side of (24). We can prove that the nested boundary $K$ matrix $K_{-}^{(1)}(u)$

$$
K_{-}^{(1)}(u)=\left(\begin{array}{ccc}
1 & 0 & 0 \\
0 & A_{-}^{(1)}(u) & B_{-}^{(1)}(u) \\
0 & C_{-}^{(1)}(u) & D_{-}^{(1)}(u)
\end{array}\right),
$$


with

$$
\begin{aligned}
& A_{-}^{(1)}(u)=\frac{q^{2 u+2}+q^{2 u}-2 q^{u+c_{a}}-2 q^{u-c_{a}+2}+q^{4}-q^{2}+2-q^{2}\left(q^{2}-1\right)\left(q^{2 u-2}-1\right) \sigma_{a}^{z}}{2\left(q^{-u+c_{a}+2}-1\right)\left(q^{u-c_{a}+2}-1\right)} \\
& B_{-}^{(1)}(u)=\frac{q\left(q^{2}-1\right)\left(q^{2 u-2}-1\right) \sigma_{a}^{-}}{\left(q^{-u+c_{a}+2}-1\right)\left(q^{u-c_{a}+2}-1\right)} \\
& C_{-}^{(1)}(u)=\frac{q\left(q^{2}-1\right)\left(q^{2 u-2}-1\right) \sigma_{a}^{+}}{\left(q^{-u+c_{a}+2}-1\right)\left(q^{u-c_{a}+2}-1\right)} \\
& D_{-}^{(1)}(u)=\frac{2 q^{2 u+2}-q^{2 u}+q^{2 u-2}-2 q^{u+c_{a}}-2 q^{u-c_{a}+2}+q^{2}+1+\left(q^{2}-1\right)\left(q^{2 u-2}-1\right) \sigma_{a}^{z}}{2\left(q^{u+c_{a}+2}-1\right)\left(q^{u-c_{a}+2}-1\right)}
\end{aligned}
$$

satisfies the nested graded reflection equation

$$
\begin{aligned}
& r_{12}(u-v) \stackrel{1}{K}(1)(u) r_{21}(u+v) \stackrel{2}{K}{ }_{-}^{(1)}(v) \\
& =\stackrel{2}{K}_{-}^{(1)}(v) r_{12}(u+v) \stackrel{1}{K} \stackrel{(1)}{-}(u) r_{21}(u-v) .
\end{aligned}
$$

Furthermore, the nested boundary $K$-matrix $K_{+}^{(1)}(u)$

$$
K_{+}^{(1)}(u)=\left(\begin{array}{ccc}
1 & 0 & 0 \\
0 & A_{+}^{(1)}(u) & B_{+}^{(1)}(u) \\
0 & C_{+}^{(1)}(u) & D_{+}^{(1)}(u)
\end{array}\right)
$$

with

$$
\begin{aligned}
& A_{+}^{(1)}(u)=-\frac{q^{u+c_{b}}\left(q^{2 u+2}-2 q^{u-c_{b}+4}-2 q^{u+c_{b}}+q^{2 u}+2 q^{4}-q^{2}+1-\left(q^{2 u}-1\right)\left(q^{2}-1\right) \sigma_{b}^{z}\right)}{2\left(q^{u+4}-q^{c_{b}}\right)\left(q^{u}-q^{c_{b}}\right)} \\
& B_{+}^{(1)}(u)=-\frac{q^{u+c_{b}}\left(q^{2 u}-1\right)\left(q^{2}-1\right) \sigma_{b}^{-}}{\left(q^{u+4}-q^{c_{b}}\right)\left(q^{u}-q^{c_{b}}\right)} \\
& C_{+}^{(1)}(u)=-\frac{q^{u+c_{b}}\left(q^{2 u}-1\right)\left(q^{2}-1\right) \sigma_{b}^{+}}{\left(q^{u+4}-q^{c_{b}}\right)\left(q^{u}-q^{c_{b}}\right)} \\
& D_{+}^{(1)}(u)=-\frac{q^{u+c_{b}}\left(q^{2 u+2}-q^{2 u}+2 q^{2 u-2}-2 q^{u-c_{b}+2}-2 q^{u+c_{b}-2}+q^{2}+1+\left(q^{2 u}-1\right)\left(q^{2}-1\right) \sigma_{b}^{z}\right)}{2\left(q^{u+4}-q^{c_{b}}\right)\left(q^{u}-q^{c_{b}}\right)}
\end{aligned}
$$

is a solution of the nested graded reflection equation

$$
\begin{aligned}
r_{21}^{s t_{1} i s t_{2}}(-u+v) \stackrel{1}{K}_{+}^{(1)^{s t_{1}}}(u)\left\{\left[r_{21}^{s t_{1}}(u+v)\right]^{-1}\right\}^{i s t_{2}} \stackrel{2}{K}_{+}^{(1)^{i s t_{2}}}(v) \\
=\stackrel{2}{K}_{+}^{(1)}{ }^{i s t_{2}}(v)\left\{\left[r_{12}^{i s t_{2}}(u+v)\right]^{-1}\right\}^{s t_{1}} \stackrel{1}{K}_{+}^{(1)^{s t_{1}}}(u) r_{12}^{s t_{1} i s t_{2}}(-u+v) .
\end{aligned}
$$

For the $R$-matrix (3) of the one-dimensional $q$-deformed supersymmetric extended Hubbard model, choosing the pseudovacuum $|0\rangle=(1,0,0,0)^{T}$, we have

$$
\begin{array}{ll}
T_{11}(u)|0\rangle=|0\rangle, & T_{d d}(u)|0\rangle=\left(\frac{q\left(q^{u}-1\right)}{q^{u}-q^{2}}\right)^{L}|0\rangle, \\
T_{1 d}(u)|0\rangle \neq 0, & T_{d b}(u)|0\rangle=0, \quad T_{d 1}(u)|0\rangle=0, \\
\tilde{T}_{11}(u)|0\rangle=|0\rangle, & \tilde{T}_{d d}(u)|0\rangle=\left(\frac{q\left(q^{u}-1\right)}{q^{u}-q^{2}}\right)^{L}|0\rangle, \\
\tilde{T}_{1 d}(u)|0\rangle \neq 0, & \tilde{T}_{d b}(u)|0\rangle=0, \quad \tilde{T}_{d 1}(u)|0\rangle=0,
\end{array}
$$


where $d \neq b, \quad d, b=2,3,4$, and

$$
\begin{aligned}
& T_{21}(u) \tilde{T}_{12}(u)|0\rangle=\frac{q^{2 u}\left(1-q^{2}\right)}{q^{2 u}-q^{2}}\left[\tilde{T}_{11}(u) T_{11}(u)-T_{22}(u) \tilde{T}_{22}(u)\right]|0\rangle, \\
& T_{\alpha 1}(u) \tilde{T}_{1 \alpha}(u)|0\rangle=0, \quad \alpha=3,4 \\
& T_{\alpha 1}(u) \tilde{T}_{1 \beta}(u)|0\rangle=0, \quad \alpha \neq \beta .
\end{aligned}
$$

Then we have

$$
\begin{aligned}
\mathcal{A}(u)|0\rangle & =T_{1 \alpha}(u) K_{-}(u)_{\alpha \beta} \tilde{T}_{\beta 1}(u)|0\rangle=|0\rangle \\
\mathcal{C}_{d}(u)|0\rangle & =T_{d a}(u) K_{-}(u)_{\alpha \beta} \tilde{T}_{\beta 1}(u)|0\rangle=0, \quad \mathcal{B}_{d}(u)|0\rangle \neq 0 \\
\check{\mathcal{D}}_{d b}(u)|0\rangle & =T_{d \alpha}(u) K_{-}(u)_{\alpha \beta} \tilde{T}_{\beta b}(u)|0\rangle=\left(\frac{q\left(q^{u}-1\right)}{q^{u}-q^{2}}\right)^{2 L} K_{-}(u)_{d b}|0\rangle \\
\check{\mathcal{D}}_{d d}(u)|0\rangle & =T_{d d}(u)\left(K_{-}(u)_{d d}-\frac{q^{2 u}\left(1-q^{2}\right)}{q^{2 u}-q^{2}}\right) \tilde{T}_{d d}(u)|0\rangle+\frac{q^{2 u}\left(1-q^{2}\right)}{q^{2 u}-q^{2}}|0\rangle .
\end{aligned}
$$

Here

$$
K_{-}(u)_{d \alpha}(u)-\frac{q^{2 u}\left(1-q^{2}\right)}{q^{2 u}-q^{2}}=\frac{q^{2}\left(q^{2 u}-1\right)}{q^{2 u}-q^{2}} K_{-}(u-1)_{d \alpha}, \quad \alpha=d, b
$$

satisfying the reflection equation (11) for the reduced problem. Letting $\check{\mathcal{D}}_{b d}(u)=\mathcal{D}_{b d}(u)-$ $\left(q^{2 u}\left(1-q^{2}\right) /\left(q^{2 u}-q^{2}\right)\right) \delta_{b d} \mathcal{A}(u)$, we have

$$
\begin{aligned}
& \check{\mathcal{D}}_{d d}(u)|0\rangle=\left(K_{-}(u)_{d d}-\frac{q^{2 u}\left(1-q^{2}\right)}{q^{2 u}-q^{2}}\right)\left(\frac{q\left(q^{u}-1\right)}{q^{u}-q^{2}}\right)^{2 L}|0\rangle, \\
& \check{\mathcal{D}}_{d b}(u)|0\rangle=K_{-}(u)_{d b}\left(\frac{q\left(q^{u}-1\right)}{q^{u}-q^{2}}\right)^{2 L}|0\rangle .
\end{aligned}
$$

Our calculations rely heavily on a variant of the (graded) Yang-Baxter relations (10) with the $R$-matrix (3),

$$
\stackrel{1}{T}(u) R(2 u) \stackrel{2}{\widetilde{T}}(u)=\stackrel{2}{\widetilde{T}}(u) R(2 u) \stackrel{1}{T}(u)
$$

on use of the following relations:

$$
\begin{gathered}
\left(q^{2 u}-q^{2}\right) T_{21}(u) \tilde{T}_{12}(u)+q^{2 u}\left(1-q^{2}\right) T_{22}(u) \tilde{T}_{22}(u)+q^{2 u}\left(1-q^{2}\right) T_{23}(u) \tilde{T}_{32}(u) \\
+q^{2 u}\left(1-q^{2}\right) T_{24}(u) \tilde{T}_{42}(u) \\
=q^{2 u}\left(1-q^{2}\right) \tilde{T}_{11}(u) T_{11}(u)+\left(q^{2 u}-1\right) \tilde{T}_{12}(u) T_{21}(u)+\left(1-q^{2}\right) \tilde{T}_{13}(u) T_{31}(u) \\
+\left(1-q^{2}\right) \tilde{T}_{14}(u) T_{41}(u), \\
\left(q^{2 u}-q^{2}\right) T_{21}(u) \tilde{T}_{13}(u)+q^{2 u}\left(1-q^{2}\right) T_{22}(u) \tilde{T}_{23}(u)+q^{2 u}\left(1-q^{2}\right) T_{23}(u) \tilde{T}_{33}(u) \\
+q^{2 u}\left(1-q^{2}\right) T_{24}(u) \tilde{T}_{43}(u)
\end{gathered}
$$




$$
\begin{aligned}
& =q\left(q^{2 u}-1\right) \tilde{T}_{13}(u) T_{22}(u), \\
& \left(q^{2 u}-q^{2}\right) T_{21}(u) \tilde{T}_{14}(u)+q^{2 u}\left(1-q^{2}\right) T_{22}(u) \tilde{T}_{24}(u)+q^{2 u}\left(1-q^{2}\right) T_{23}(u) \tilde{T}_{34}(u) \\
& +q^{2 u}\left(1-q^{2}\right) T_{24}(u) \widetilde{T}_{44}(u) \\
& =q\left(q^{2 u}-1\right) \tilde{T}_{14}(u) T_{22}(u), \\
& \left(1-q^{2}\right) T_{31}(u) \tilde{T}_{12}(u)+\left(q^{2 u}-q^{2}\right) T_{32}(u) \tilde{T}_{22}(u) \\
& +q^{2 u}\left(1-q^{2}\right) T_{33}(u) \tilde{T}_{32}(u)+q^{2 u}\left(1-q^{2}\right) T_{34}(u) \tilde{T}_{42}(u) \\
& =q\left(q^{2 u}-1\right) \tilde{T}_{22}(u) T_{32}(u), \\
& \left(1-q^{2}\right) T_{31}(u) \tilde{T}_{13}(u)+\left(q^{2 u}-q^{2}\right) T_{32}(u) \tilde{T}_{23}(u)+q^{2 u}\left(1-q^{2}\right) T_{33}(u) \tilde{T}_{33}(u) \\
& +q^{2 u}\left(1-q^{2}\right) T_{34}(u) \tilde{T}_{43}(u) \\
& =q\left(q^{2 u}-1\right) \tilde{T}_{21}(u) T_{12}(u)+q^{2 u}\left(1-q^{2}\right) \tilde{T}_{22}(u) T_{22}(u)-\left(q^{2 u+2}-1\right) \tilde{T}_{23}(u) T_{32}(u) \\
& +\left(1-q^{2}\right) \tilde{T}_{24}(u) T_{42}(u) \text {, } \\
& \left(1-q^{2}\right) T_{31}(u) \tilde{T}_{14}(u)+\left(q^{2 u}-q^{2}\right) T_{32}(u) \tilde{T}_{24}(u)+q^{2 u}\left(1-q^{2}\right) T_{33}(u) \tilde{T}_{34}(u) \\
& +q^{2 u}\left(1-q^{2}\right) T_{34}(u) \tilde{T}_{44}(u) \\
& =-q\left(q^{2 u}-1\right) \tilde{T}_{24}(u) T_{32}(u), \\
& \left(1-q^{2}\right) T_{41}(u) \tilde{T}_{12}(u)+\left(1-q^{2}\right) T_{42}(u) \tilde{T}_{22}(u)-\left(q^{2 u+2}-1\right) T_{43}(u) \tilde{T}_{32}(u) \\
& +q^{2 u}\left(1-q^{2}\right) T_{44}(u) \tilde{T}_{42}(u) \\
& =-q\left(q^{2 u}-1\right) \tilde{T}_{32}(u) T_{43}(u), \\
& \left(1-q^{2}\right) T_{41}(u) \tilde{T}_{13}(u)+\left(1-q^{2}\right) T_{42}(u) \tilde{T}_{23}(u)\left(q^{2 u+2}-1\right) T_{43}(u) \tilde{T}_{33}(u) \\
& +q^{2 u}\left(1-q^{2}\right) T_{44}(u) \widetilde{T}_{43}(u) \\
& =-q\left(q^{2 u}-1\right) \tilde{T}_{33}(u) T_{43}(u), \\
& \left(1-q^{2}\right) T_{41}(u) \tilde{T}_{13}(u)+\left(1-q^{2}\right) T_{42}(u) \tilde{T}_{23}(u)-\left(q^{2 u+2}-1\right) T_{43}(u) \tilde{T}_{33}(u) \\
& +q^{2 u}\left(1-q^{2}\right) T_{44}(u) \tilde{T}_{43}(u) \\
& =q^{2 u}\left(1-q^{2}\right) \tilde{T}_{31}(u) T_{13}(u)+q^{2 u}\left(1-q^{2}\right) \tilde{T}_{32}(u) T_{23}(u)+q^{2 u}\left(1-q^{2}\right) \tilde{T}_{33}(u) T_{33}(u) \\
& -\left(q^{2 u+2}-1\right) \tilde{T}_{34}(u) T_{43}(u) \text {. }
\end{aligned}
$$

Implementing the change $u \rightarrow u-1$ with respect to the original problem, one may check that the nested boundary $K$ matrices (30) and (32) still satisfy the reflection equations (31) and (33) for the reduced problem. After some algebra, the nested transfer matrix $\tau^{(1)}(u)$ may be recognised as that for the one-dimensional $q$-deformed supersymmetric $t-J$ model with boundary Kondo impurities, which has been diagonalised in $[8,11]$. 
Here we merely give the final result,

$$
\begin{aligned}
\Lambda^{(1)}\left(u ;\left\{u_{j}\right\}\right)= & \frac{\left(q^{2 u}-1\right)\left(q^{u+2}-q^{c_{b}}\right)\left(q^{u}-q^{c_{b}+2}\right)}{\left(q^{2 u-2}-q^{2}\right)\left(q^{u}-q^{c_{b}}\right)\left(q^{u+4}-q^{c_{b}}\right)} \prod_{k=1}^{M_{1}} \frac{\left(q^{u+u_{k}^{(1)}-2}-1\right)\left(q^{u-u_{k}^{(1)}+2}-1\right)}{\left(q^{u+u_{k}^{(1)}-2}-q^{2}\right)\left(q^{u-u_{k}^{(1)}}-1\right)} \\
& -\frac{q^{2}\left(q^{2 u-2}-1\right)}{q^{2 u-2}-q^{2}} \prod_{j=1}^{N} \frac{\left(q^{u+u_{j}-2}-1\right)\left(q^{u-u_{j}}-1\right)}{\left(q^{u+u_{j}-2}-q^{2}\right)\left(q^{u-u_{j}-2}-1\right)} \\
& \prod_{k=1}^{M_{1}} \frac{\left(q^{u+u_{k}^{(1)}-2}-1\right)\left(q^{u-u_{k}^{(1)}+2}-1\right)}{\left(q^{u+u_{k}^{(1)}-2}-q^{2}\right)\left(q^{u-u_{k}^{(1)}}-1\right)} \Lambda^{(2)}\left(u ;\left\{u_{j}\right\} ;\left\{u_{k}^{(1)}\right\}\right),
\end{aligned}
$$

provided the parameters $\left\{u_{k}^{(1)}\right\}$ satisfy

$$
\begin{gathered}
\frac{\left(q^{2 u_{k}^{(1)}}-1\right)\left(q^{u_{k}^{(1)}+2}-q^{c_{b}}\right)\left(q^{u_{k}^{(1)}}-q^{c_{b}+2}\right)}{\left(q^{2 u_{k}^{(1)}}-q^{2}\right)\left(q^{u_{k}^{(1)}}-q^{c_{b}}\right)\left(q^{u_{k}^{(1)}+4}-q^{c_{b}}\right)} \prod_{j=1}^{N} \frac{\left(q^{u_{k}^{(1)}+u_{j}-2}-q^{2}\right)\left(q^{u_{k}^{(1)}-u_{j}}-q^{2}\right)}{q^{2}\left(q_{k}^{u_{k}^{(1)}+u_{j}-2}-1\right)\left(q^{u_{k}^{(1)}-u_{j}}-1\right)} \\
=\Lambda^{(2)}\left(u_{k}^{(1)} ;\left\{u_{i}\right\} ;\left\{u_{l}^{(1)}\right\}\right) .
\end{gathered}
$$

Here $\Lambda^{(2)}\left(u ;\left\{u_{j}\right\},\left\{u_{k}^{(1)}\right\}\right)$ is the eigenvalue of the nested transfer matrix $\tau^{(2)}(u)$ for the $\left(M_{2}+2\right)$-site $X X Z$ spin-1/2 open chain,

$$
\begin{aligned}
& \Lambda^{(2)}\left(u ;\left\{u_{j}\right\} ;\left\{u_{k}^{(1)}\right\}\right)=\frac{\left(q^{u+2}-q^{c_{b}}\right)\left(q^{u}-q^{c_{b}+2}\right)}{\left(q^{u}-q^{c_{b}}\right)\left(q^{u+4}-q^{c_{b}}\right)} \\
& \prod_{\gamma=a, b} \frac{q^{u+c_{\gamma}-1}-1}{q^{u-2}-q^{c_{\gamma}+1}}\left\{\frac{q^{2 u}-1}{q^{2}\left(q^{2 u-2}-1\right)} \prod_{l=1}^{M_{2}} \frac{\left(q^{u-u_{l}^{(2)}}-q^{2}\right)\left(q^{u+u_{i}^{(2)}-2}-q^{2}\right)}{q^{2}\left(q^{u-u_{l}^{(2)}}-1\right)\left(q^{u+u_{l}^{(2)}-2}-1\right)}\right. \\
& +\frac{q^{2 u-2}-q^{2}}{q^{2}\left(q^{2 u-2}-1\right)} \prod_{\gamma=a, b} \frac{\left(q^{u+c_{\gamma}-1}-q^{2}\right)\left(q^{u-c_{\gamma}+1}-q^{2}\right)}{q^{2}\left(q^{u+c_{\gamma}-1}-1\right)\left(q^{u-c_{\gamma}+1}-1\right)} \prod_{k=1}^{M_{1}} \frac{\left(q^{u+u_{\alpha}^{(1)}-2}-q^{2}\right)\left(q^{u-u_{k}^{(1)}+2}-q^{2}\right)}{q^{2}\left(q^{u+u_{k}^{(1)}-2}-1\right)\left(q^{u-u_{k}^{(1)}+2}-1\right)} \\
& \left.\prod_{l=1}^{M_{2}} \frac{q^{2}\left(q^{u-u_{l}^{(2)}+2}-1\right)\left(q^{u+u_{l}^{(2)}}-1\right)}{\left(q^{u-u_{l}^{(2)}+2}-q^{2}\right)\left(q^{u+u_{l}^{(2)}}-q^{2}\right)}\right\}
\end{aligned}
$$

provided the parameters $\left\{u_{l}^{(2)}\right\}$ satisfy

$$
\begin{aligned}
& \prod_{\gamma=a, b} \frac{\left(q^{u_{l}^{(2)}+c_{\gamma}-1}-q^{2}\right)\left(q^{u_{i}^{(2)}-c_{\gamma}+1}-q^{2}\right)}{q^{2}\left(q_{i}^{u_{l}^{(2)}+c_{\gamma}-1}-1\right)\left(q^{u_{l}^{(2)}-c_{\gamma}+1}-1\right)} \prod_{k=1}^{M_{1}} \frac{\left(q^{u_{i}^{(2)}+u_{k}^{(1)}-2}-q^{2}\right)\left(q^{u_{l}^{(2)}-u_{k}^{(1)}+2}-q^{2}\right)}{q^{2}\left(q^{u_{i}^{(2)}+u_{k}^{(1)}-2}-1\right)\left(q^{u_{l}^{(2)}-u_{k}^{(1)}+2}-1\right)} \\
& \quad=\prod_{\substack{p=1 \\
p \neq l}}^{M_{2}} \frac{\left(q^{u_{i}^{(2)}-u_{p}^{(2)}+2}-q^{4}\right)\left(q^{u_{l}^{(2)}+u_{p}^{(2)}}-q^{4}\right)}{q^{4}\left(q_{i}^{u_{i}^{(2)}-u_{p}^{(2)}+2}-1\right)\left(q^{u_{l}^{(2)}+u_{p}^{(2)}}-1\right)}
\end{aligned}
$$


After a shift of the parameters $u_{j} \rightarrow u_{j}+1, u_{k}^{(1)} \rightarrow u_{k}^{(1)}+2, u_{l}^{(2)} \rightarrow u_{l}^{(2)}+1$, and $c_{\gamma} \rightarrow c_{\gamma}+1$, the Bethe ansatz equations (29) and (40) and (42) may be rewritten as follows:

$$
\begin{aligned}
& \left(\frac{q\left(q^{u_{j}-1}-1\right)}{q^{u_{j}+1}-1}\right)^{2 L} \prod_{\substack{i=1 \\
i \neq j}}^{N} \frac{\left(q^{u_{j}+u_{i}+2}-1\right)\left(q^{u_{j}-u_{i}+2}-1\right)}{q^{4}\left(q^{u_{j}+u_{i}-2}-1\right)\left(q^{u_{j}-u_{i}-2}-1\right)} \\
& =\prod_{k=1}^{M_{1}} \frac{\left(q^{u_{j}+u_{k}^{(1)}+1}-1\right)\left(q^{u_{j}-u_{k}^{(1)}+1}-1\right)}{q^{2}\left(q^{u_{j}+u_{k}^{(1)}-1}-1\right)\left(q^{u_{j}-u_{k}^{(1)}-1}-1\right)} \\
& \prod_{\gamma=a, b} \frac{q^{u_{k}^{(1)}+c_{\gamma}+2}-1}{q^{c_{\gamma}+2}\left(q^{u_{k}^{(1)}-c_{\gamma}-2}-1\right)} \prod_{j=1}^{N} \frac{\left(q^{u_{k}^{(1)}-u_{j}+1}-1\right)\left(q^{u_{k}^{(1)}+u_{j}+1}-1\right)}{q^{2}\left(q^{u_{k}^{(1)}-u_{j}-1}-1\right)\left(q^{u_{k}^{(1)}+u_{j}-1}-1\right)} \\
& =\prod_{l=1}^{M_{2}} \frac{\left(q^{u_{k}^{(1)}-u_{l}^{(2)}+1}-1\right)\left(q^{u_{k}^{(1)}+u_{i}^{(2)}+1}-1\right)}{q^{2}\left(q^{u_{k}^{(1)}-u_{i}^{(2)}-1}-1\right)\left(q^{u_{k}^{(1)}+u_{i}^{(2)}-1}-1\right)} \\
& \prod_{\gamma=a, b} \frac{\left(q^{u_{l}^{(2)}+c_{\gamma}+1}-q^{2}\right)\left(q^{u_{l}^{(2)}-c_{\gamma}+1}-q^{2}\right)}{q^{2}\left(q_{l}^{u_{l}^{(2)}+c_{\gamma}+1}-1\right)\left(q^{u_{l}^{(2)}-c_{\gamma}+1}-1\right)} \prod_{k=1}^{M_{1}} \frac{q^{2}\left(q^{u_{l}^{(2)}+u_{k}^{(1)}-1}-1\right)\left(q^{u_{l}^{(2)}-u_{k}^{(1)}-1}-1\right)}{\left(q^{u_{l}^{(2)}+u_{k}^{(1)}+1}-1\right)\left(q^{u_{l}^{(2)}-u_{k}^{(1)}+1}-1\right)} \\
& =\prod_{\substack{p=1 \\
p \neq l}}^{M_{2}} \frac{q^{4}\left(q^{u_{l}^{(1)}-u_{p}^{(2)}-2}-1\right)\left(q^{u_{l}^{(2)}+u_{p}^{(2)}-2}-1\right)}{\left(q_{i}^{u_{l}^{(1)}-u_{p}^{(2)}+2}-1\right)\left(q^{u_{l}^{(2)}+u_{p}^{(2)}+2}-1\right)}
\end{aligned}
$$

or

$$
\begin{gathered}
\left(\frac{\sinh \left(\gamma\left(u_{j}-1\right) / 2\right)}{\sinh \left(\gamma\left(u_{j}+1\right) / 2\right)}\right)^{2 L} \prod_{\substack{i=1 \\
i \neq j}}^{N} \frac{\sinh \left(\gamma\left(u_{j}+u_{i}+2\right) / 2\right) \sinh \left(\gamma\left(u_{j}-u_{i}+2\right) / 2\right)}{\sinh \left(\gamma\left(u_{j}+u_{i}-2\right) / 2\right) \sinh \left(\gamma\left(u_{j}-u_{i}-2\right) / 2\right)} \\
=\prod_{k=1}^{M_{1}} \frac{\sinh \left(\gamma\left(u_{j}+u_{k}^{(1)}+1\right) / 2\right) \sinh \left(\gamma\left(u_{j}-u_{k}^{(1)}+1\right) / 2\right)}{\sinh \left(\gamma\left(u_{j}+u_{k}^{(1)}-1\right) / 2\right) \sinh \left(\gamma\left(u_{j}-u_{k}^{(1)}-1\right) / 2\right)} \\
\prod_{\alpha=a, b} \frac{\sinh \left(\gamma\left(u_{k}^{(1)}+c_{\alpha}+2\right) / 2\right)}{\sinh \left(\gamma\left(u_{k}^{(1)}-c_{\alpha}-2\right) / 2\right)} \prod_{j=1}^{N} \frac{\sinh \left(\gamma\left(u_{k}^{(1)}-u_{j}+1\right) / 2\right) \sinh \left(\gamma\left(u_{k}^{(1)}+u_{j}+1\right) / 2\right)}{\left.\sinh \left(u_{k}^{(1)}-u_{j}-1\right) / 2\right) \sinh \left(\gamma\left(u_{k}^{(1)}+u_{j}-1\right) / 2\right)} \\
=\prod_{l=1}^{M_{2}} \frac{\sinh \left(\gamma\left(u_{k}^{(1)}-u_{l}^{(2)}+1\right) / 2\right) \sinh \left(\gamma\left(u_{k}^{(1)}+u_{l}^{(2)}+1\right) / 2\right)}{\sinh \left(\gamma\left(u_{k}^{(1)}-u_{l}^{(2)}-1\right) / 2\right) \sinh \left(\gamma\left(u_{k}^{(1)}+u_{l}^{(2)}-1\right) / 2\right)}, \\
\prod_{\alpha=a, b} \frac{\sinh \left(\gamma\left(u_{l}^{(2)}+c_{\alpha}-1\right) / 2\right) \sinh \left(\gamma\left(u_{l}^{(2)}-c_{\alpha}-1\right) / 2\right)}{\sinh \left(\gamma\left(u_{l}^{(2)}+c_{\alpha}+1\right) / 2\right) \sinh \left(\gamma\left(u_{l}^{(2)}-c_{\alpha}+1\right) / 2\right)} \\
\quad \times \prod_{k=1}^{M_{1}} \frac{\sinh \left(\gamma\left(u_{l}^{(2)}+u_{k}^{(1)}-1\right) / 2\right) \sinh \left(\gamma\left(u_{l}^{(2)}-u_{k}^{(1)}-1\right) / 2\right)}{\sinh \left(\gamma\left(u_{l}^{(2)}+u_{k}^{(1)}+1\right) / 2\right) \sinh \left(\gamma\left(u_{l}^{(2)}-u_{k}^{(1)}+1\right) / 2\right)}
\end{gathered}
$$




$$
=\prod_{\substack{p=1 \\ p \neq l}}^{M_{2}} \frac{\sinh \left(\gamma\left(u_{l}^{(2)}-u_{p}^{(2)}-2\right) / 2\right) \sinh \left(\gamma\left(u_{l}^{(2)}+u_{p}^{(2)}-2\right) / 2\right)}{\sinh \left(\gamma\left(u_{l}^{(2)}-u_{p}^{(2)}+2\right) / 2\right) \sinh \left(\gamma\left(u_{l}^{(2)}+u_{p}^{(2)}+2\right) / 2\right)}
$$

with $q=e^{\gamma}$. The corresponding energy eigenvalue of the model is given by

$$
E=-\sum_{j=1}^{n} \frac{4}{\sinh \left(\gamma\left(u_{j}-1\right) / 2\right) \sinh \left(\gamma\left(u_{j}+1\right) / 2\right)} .
$$

(modulo an unimportant additive constant, which we drop).

Similarly we can apply the algebraic Bethe ansatz method for the second coupling (5). The Bethe ansatz equations are

$$
\begin{gathered}
\left(\frac{\sinh \left(\gamma\left(u_{j}-1\right) / 2\right)}{\sinh \left(\gamma\left(u_{j}+1\right) / 2\right)}\right)^{2 L} \prod_{\substack{i=1 \\
i \neq j}}^{N} \frac{\sinh \left(\gamma\left(u_{j}+u_{i}+2\right) / 2\right) \sinh \left(\gamma\left(u_{j}-u_{i}+2\right) / 2\right)}{\sinh \left(\gamma\left(u_{j}+u_{i}-2\right) / 2\right) \sinh \left(\gamma\left(u_{j}-u_{i}-2\right) / 2\right)} \\
=\prod_{k=1}^{M_{1}} \frac{\sinh \left(\gamma\left(u_{j}+u_{k}^{(1)}+1\right) / 2\right) \sinh \left(\gamma\left(u_{j}-u_{k}^{(1)}+1\right) / 2\right)}{\sinh \left(\gamma\left(u_{j}+u_{k}^{(1)}-1\right) / 2\right) \sinh \left(\gamma\left(u_{j}-u_{k}^{(1)}-1\right) / 2\right)} \\
\prod_{\alpha=a, b} \frac{\sinh \left(\gamma\left(u_{k}^{(1)}+c_{\alpha}+4\right) / 2\right)}{\sinh \left(\gamma\left(u_{k}^{(1)}-c_{\alpha}-4\right) / 2\right)} \prod_{j=1}^{N} \frac{\sinh \left(\gamma\left(u_{k}^{(1)}-u_{j}-1\right) / 2\right) \sinh \left(\gamma\left(u_{k}^{(1)}+u_{j}-1\right) / 2\right)}{\sinh \left(\gamma\left(u_{k}^{(1)}-u_{j}+1\right) / 2\right) \sinh \left(\gamma\left(u_{k}^{(1)}+u_{j}+1\right) / 2\right)} \\
=\prod_{l=1}^{M_{2}} \frac{\sinh \left(\gamma\left(u_{k}^{(1)}-u_{l}^{(2)}+1\right) / 2\right) \sinh \left(\gamma\left(u_{k}^{(1)}+u_{l}^{(2)}+1\right) / 2\right)}{\sinh \left(\gamma\left(u_{k}^{(1)}-u_{l}^{(2)}-1\right) / 2\right) \sinh \left(\gamma\left(u_{k}^{(1)}+u_{l}^{(2)}-1\right) / 2\right)} \\
\quad \times \prod_{\substack{\delta=1 \\
\delta \neq k}}^{M_{1}} \frac{\sinh \left(\gamma\left(u_{k}^{(1)}-u_{\delta}^{(1)}-2\right) / 2\right) \sinh \left(\gamma\left(u_{k}^{(1)}+u_{\delta}^{(1)}-2\right) / 2\right)}{\sinh \left(\gamma\left(u_{k}^{(1)}-u_{\delta}^{(1)}+2\right) / 2\right) \sinh \left(\gamma\left(u_{k}^{(1)}+u_{\delta}^{(1)}+2\right) / 2\right)} \\
\prod_{\alpha=a, b} \frac{\sinh \left(\gamma\left(u_{l}^{(2)}+c_{\alpha}+3\right) / 2\right) \sinh \left(\gamma\left(u_{l}^{(2)}-c_{\alpha}-1\right) / 2\right)}{\sinh \left(\gamma\left(u_{l}^{(2)}-c_{\alpha}-3\right) / 2\right) \sinh \left(\gamma\left(u_{l}^{(2)}+c_{\alpha}+1\right) / 2\right)} \\
\quad \times \prod_{k=1}^{M_{1}} \frac{\sinh \left(\gamma\left(u_{l}^{(2)}+u_{k}^{(1)}-1\right) / 2\right) \sinh \left(\gamma\left(u_{l}^{(2)}-u_{k}^{(1)}-1\right) / 2\right)}{\sinh \left(\gamma\left(u_{l}^{(2)}+u_{k}^{(1)}+1\right) / 2\right) \sinh \left(\gamma\left(u_{l}^{(2)}-u_{k}^{(1)}+1\right) / 2\right)} \\
=\prod_{\substack{p=1 \\
p \neq l}}^{M_{2}} \frac{\sinh \left(\gamma\left(u_{l}^{(2)}-u_{p}^{(2)}+2\right) / 2\right) \sinh \left(\gamma\left(u_{l}^{(2)}+u_{p}^{(2)}+2\right) / 2\right)}{\sinh \left(\gamma\left(u_{l}^{(2)}-u_{p}^{(2)}-2\right) / 2\right) \sinh \left(\gamma\left(u_{l}^{(2)}+u_{p}^{(2)}-2\right) / 2\right)}
\end{gathered}
$$

with the corresponding energy eigenvalue $E$ of the model in (45).

We can also apply the algebraic Bethe ansatz method for the third coupling (8). The Bethe ansatz equations are

$$
\left(\frac{\sinh \left(\gamma\left(u_{j}+1\right) / 2\right)}{\sinh \left(\gamma\left(u_{j}-1\right) / 2\right)}\right)^{2 L} \prod_{\substack{i=1 \\ i \neq j}}^{N} \frac{\sinh \left(\gamma\left(u_{j}+u_{i}+2\right) / 2\right) \sinh \left(\gamma\left(u_{j}-u_{i}+2\right) / 2\right)}{\sinh \left(\gamma\left(u_{j}+u_{i}-2\right) / 2\right) \sinh \left(\gamma\left(u_{j}-u_{i}-2\right) / 2\right)}
$$




$$
\begin{aligned}
& =\prod_{k=1}^{M_{1}} \frac{\sinh \left(\gamma\left(u_{j}+u_{k}^{(1)}+1\right) / 2\right) \sinh \left(\gamma\left(u_{j}-u_{k}^{(1)}+1\right) / 2\right)}{\sinh \left(\gamma\left(u_{j}+u_{k}^{(1)}-1\right) / 2\right) \sinh \left(\gamma\left(u_{j}-u_{k}^{(1)}-1\right) / 2\right)} \\
& \prod_{\alpha=a, b} \frac{\sinh \left(\gamma\left(u_{k}^{(1)}+c_{\alpha}+2\right) / 2\right)}{\sinh \left(\gamma\left(u_{k}^{(1)}-c_{\alpha}-2\right) / 2\right)} \prod_{j=1}^{N} \frac{\sinh \left(\gamma\left(u_{k}^{(1)}-u_{j}-1\right) / 2\right) \sinh \left(\gamma\left(u_{k}^{(1)}+u_{j}-1\right) / 2\right)}{\sinh \left(\gamma\left(u_{k}^{(1)}-u_{j}+1\right) / 2\right) \sinh \left(\gamma\left(u_{k}^{(1)}+u_{j}+1\right) / 2\right)} \\
& =\prod_{l=1}^{M_{2}} \frac{\sinh \left(\gamma\left(u_{k}^{(1)}-u_{l}^{(2)}+1\right) / 2\right) \sinh \left(\gamma\left(u_{k}^{(1)}+u_{l}^{(2)}+1\right) / 2\right)}{\sinh \left(\gamma\left(u_{k}^{(1)}-u_{l}^{(2)}-1\right) / 2\right) \sinh \left(\gamma\left(u_{k}^{(1)}+u_{l}^{(2)}-1\right) / 2\right)} \\
& \times \prod_{\substack{\delta=1 \\
\delta \neq k}}^{M_{1}} \frac{\sinh \left(\gamma\left(u_{k}^{(1)}-u_{\delta}^{(1)}-2\right) / 2\right) \sinh \left(\gamma\left(u_{k}^{(1)}+u_{\delta}^{(1)}-2\right) / 2\right)}{\sinh \left(\gamma\left(u_{k}^{(1)}-u_{\delta}^{(1)}+2\right) / 2\right) \sinh \left(\gamma\left(u_{k}^{(1)}+u_{\delta}^{(1)}+2\right) / 2\right)} \\
& \prod_{\alpha=a, b} \frac{\sinh \left(\gamma\left(u_{l}^{(2)}+c_{\alpha}+1\right) / 2\right) \sinh \left(\gamma\left(u_{l}^{(2)}-c_{\alpha}+1\right) / 2\right)}{\sinh \left(\gamma\left(u_{l}^{(2)}-c_{\alpha}-1\right) / 2\right) \sinh \left(\gamma\left(u_{l}^{(2)}+c_{\alpha}-1\right) / 2\right)} \\
& \times \prod_{k=1}^{M_{1}} \frac{\sinh \left(\gamma\left(u_{l}^{(2)}+u_{k}^{(1)}+1\right) / 2\right) \sinh \left(\gamma\left(u_{l}^{(2)}-u_{k}^{(1)}+1\right) / 2\right)}{\sinh \left(\gamma\left(u_{l}^{(2)}+u_{k}^{(1)}-1\right) / 2\right) \sinh \left(\gamma\left(u_{l}^{(2)}-u_{k}^{(1)}-1\right) / 2\right)} \\
& \text { (47) }=\prod_{\substack{p=1 \\
p \neq l}}^{M_{2}} \frac{\sinh \left(\gamma\left(u_{l}^{(2)}-u_{p}^{(2)}+2\right)\right) 2 \sinh \left(\gamma\left(u_{l}^{(2)}+u_{p}^{(2)}+2\right) / 2\right)}{\sinh \left(\gamma\left(u_{l}^{(2)}-u_{p}^{(2)}-2\right) / 2\right) \sinh \left(\gamma\left(u_{l}^{(2)}+u_{p}^{(2)}-2\right) / 2\right)},
\end{aligned}
$$

with the corresponding energy eigenvalue $E$ of the model in (45).

\section{Conclusion}

In conclusion, we have studied an integrable Kondo problem describing two impurities coupled to three kinds of one-dimensional $q$-deformed extended Hubbard open chains. The quantum integrability of these systems follow from the fact that the Hamiltonian may be derived from boundary transfer matrix. Moreover, the Bethe ansatz equations are obtained by means of the algebraic Bethe ansatz approach. It should be emphasised that the boundary $K$ matrices found here are highly nontrivial, since they cannot be factorised into the product of a c-number $K$ matrix and the local monodromy matrices. Taking the limit $q \rightarrow 1$ in the Bethe ansatz equations (43), (46) and (47), we can easily obtain the Bethe ansatz equations for the one-dimensional supersymmetric $g l(2 \mid 2), s u(4)$ and $g l(3 \mid 1)$ extended Hubbard models with the boundary Kondo impurities, described when $s_{\alpha}=1 / 2$ in $[23]$.

Appendix A: Derivation of THE Non-C-Number Boundary $K$-Matrices for The $q$-Deformed Essler-Korepin-Schoutens Model With Boundary Kondo IMPURITIES

In this Appendix, we sketch the procedure for solving the graded reflection equation (11) for $K_{-}(u)$. To describe the integrable $q$-deformed Essler-Korepin-Schoutens model 
with boundary Kondo impurities, it is reasonable to assume that

$$
K_{-}(u)=\left(\begin{array}{cccc}
1 & 0 & 0 & 0 \\
0 & 1 & 0 & 0 \\
0 & 0 & \bar{A}(u) & \bar{B}(u) \\
0 & 0 & \bar{C}(u) & \bar{D}(u)
\end{array}\right)
$$

Choosing $\bar{A}(u)=F^{-1}(u) A(u), \bar{B}(u)=F^{-1}(u) B(u), \bar{C}(u)=F^{-1}(u) C(u), \bar{D}(u)$ $=F^{-1}(u) D(u)$, then

$$
K_{-}(u) \propto\left(\begin{array}{cccc}
F(u) & 0 & 0 & 0 \\
0 & F(u) & 0 & 0 \\
0 & 0 & A(u) & B(u) \\
0 & 0 & C(u) & D(u)
\end{array}\right)
$$

For the $R$-matrix (3), one may get from the equation (11), 54 functional equations, of which 14 are identities. After some algebraic analysis, together with the $U_{q}(s u(2))$ symmetry, we may assume that

$$
\begin{array}{ll}
A(u)=\alpha(u)-\beta(u) \sigma^{z}, & B(u)=\gamma(u) \sigma^{-}, \\
C(u)=\gamma(u) \sigma^{+}, & D(u)=\tilde{\alpha}(u)+\tilde{\beta}(u) \sigma^{z} .
\end{array}
$$

There are 10 equations automatically satisfied and 10 repeated equations, leaving only 20 equations to be solved:

$$
\begin{aligned}
& A(u) B(v)+B(u) D(v)=A(v) B(u)+B(v) D(u) \\
& C(u) A(v)+D(u) C(v)=C(v) A(u)+D(v) C(u) \\
& \left(q^{u_{-}}-1\right)(A(u) B(v)+B(u) D(v))=\left(q^{u_{+}}-1\right)\left(F(v) B(u)-q^{u_{-}} F(u) B(v)\right) \\
& \left(q^{u_{-}}-1\right)(A(v) B(u)+B(v) D(u))=\left(q^{u_{+}}-1\right)\left(B(u) F(v)-q^{u_{-}} B(v) F(u)\right) \\
& \left(q^{u_{-}}-1\right)(C(u) A(v)+D(u) C(v))=\left(q^{u_{+}}-1\right)\left(F(v) C(u)-q^{u_{-}} F(u) C(v)\right) \\
& \left(q^{u_{-}}-1\right)(C(v) A(u)+D(v) C(u))=\left(q^{u_{+}}-1\right)\left(C(u) F(v)-q^{u_{-}} C(v) F(u)\right) \\
& \left(q^{u_{-}}-1\right)\left(A(u) A(v)+B(u) C(v)-q^{u_{+}} F(v) F(u)\right) \\
& \quad=\left(q^{u_{+}}-1\right)\left(F(v) A(u)-q^{u_{-}} F(u) A(v)\right) \\
& \left(q^{u_{-}}-1\right)\left(A(v) A(u)+B(v) C(u)-q^{u_{+}} F(u) F(v)\right) \\
& \quad=\left(q^{u_{+}}-1\right)\left(A(u) F(v)-q^{u_{-}} A(v) F(u)\right) \\
& \left(q^{u_{-}}-1\right)\left(C(u) B(v)+D(u) D(v)-q^{u_{+}} F(v) F(u)\right) \\
& \quad=\left(q^{u_{+}}-1\right)\left(F(v) D(u)-q^{u_{-}} F(u) D(v)\right) \\
& \left(q^{u_{-}}-1\right)\left(C(v) B(u)+D(v) D(u)-q^{u_{+}} F(u) F(v)\right)
\end{aligned}
$$




$$
\begin{aligned}
& =\left(q^{u_{+}}-1\right)\left(D(u) F(v)-q^{u_{-}} D(v) F(u)\right), \\
& \left(q^{u_{-}}-1\right)\left(\left(q^{u_{+}+2}-1\right) B(u) D(v)-q^{u_{+}}\left(1-q^{2}\right) A(u) B(v)\right) \\
& =\left(q^{u_{+}}-1\right)\left(\left(q^{u_{-}+2}-1\right) D(v) B(u)+\left(1-q^{2}\right) D(u) B(v)\right), \\
& \left(q^{u-}-1\right)\left(\left(q^{u_{+}+2}-1\right) D(v) C(u)-q^{u_{+}}\left(1-q^{2}\right) C(v) A(u)\right) \\
& =\left(q^{u_{+}}-1\right)\left(\left(q^{u_{-}+2}-1\right) C(u) D(v)+\left(1-q^{2}\right) C(v) D(u)\right), \\
& \left(q^{u_{-}}-1\right)\left(\left(q^{u_{+}+2}-1\right) C(u) A(v)-\left(1-q^{2}\right) D(u) C(v)\right) \\
& =\left(q^{u_{+}}-1\right)\left(\left(q^{u_{-}+2}-1\right) A(v) C(u)+q^{u_{-}}\left(1-q^{2}\right) A(u) C(v)\right) \text {, } \\
& \left(q^{u_{-}}-1\right)\left(\left(1-q^{2}\right)\left(q^{u_{+}} A(u) A(v)-D(v) D(u)\right)-\left(q^{u_{+}+2}-1\right)(B(u) C(v)-C(v) B(u))\right) \\
& =\left(q^{u_{+}}-1\right)\left(1-q^{2}\right)\left(q^{u_{-}} D(v) A(u)-D(u) A(v)\right) \\
& \left(q^{u_{-}}-1\right)\left(\left(1-q^{2}\right)\left(q^{u_{+}} A(v) A(u)-D(u) D(v)\right)-\left(q^{u_{+}+2}-1\right)(B(v) C(u)-C(u) B(v))\right) \\
& =\left(q^{u_{+}}-1\right)\left(1-q^{2}\right)\left(q^{u_{-}} A(u) D(v)-A(v) D(u)\right) \\
& \left(q^{u++2}-1\right)\left(\left(1-q^{2}\right) C(u) A(v)+\left(q^{u-+2}-1\right) C(v) A(u)\right) \\
& =\left(1-q^{2}\right)\left(\left(q^{u_{-}+2}-1\right) D(v) C(u)+\left(1-q^{2}\right) D(u) C(v)\right) \\
& +q^{2}\left(q^{u_{+}}-1\right)\left(q^{u_{-}}-1\right) A(u) C(v) \\
& \left(q^{u_{+}+2}-1\right)\left(\left(1-q^{2}\right) A(v) B(u)+\left(q^{u_{-}+2}-1\right) A(u) B(v)\right) \\
& =\left(1-q^{2}\right)\left(\left(q^{u_{-}+2}-1\right) B(u) D(v)+\left(1-q^{2}\right) B(v) D(u)\right) \\
& +q^{2}\left(q^{u_{+}}-1\right)\left(q^{u_{-}}-1\right) B(v) A(u) \\
& \left(q^{u_{+}+2}-1\right)\left(q^{u_{-}}\left(1-q^{2}\right) B(u) D(v)+\left(q^{u_{-}+2}-1\right) B(v) D(u)\right) \\
& =q^{u_{+}}\left(1-q^{2}\right)\left(\left(q^{u_{-}+2}-1\right) A(v) B(u)+q^{u_{-}}\left(1-q^{2}\right) A(u) B(v)\right) \\
& +q^{2}\left(q^{u_{+}}-1\right)\left(q^{u_{-}}-1\right) D(u) B(v) \\
& \left(q^{u_{+}+2}-1\right)\left(q^{u_{-}}\left(1-q^{2}\right) D(v) C(u)+\left(q^{u_{-}+2}-1\right) D(u) C(v)\right) \\
& =q^{u+}\left(1-q^{2}\right)\left(\left(q^{u_{-}+2}-1\right) C(u) A(v)+q^{u_{-}}\left(1-q^{2}\right) C(v) A(u)\right) \\
& +q^{2}\left(q^{u_{+}}-1\right)\left(q^{u_{-}}-1\right) C(v) D(u),
\end{aligned}
$$

with $u_{+}=u+v, u_{-}=u-v$. Substituting (50) into these equations, we find that all these equations are reduced to the following 11 equations:

$$
\begin{gathered}
(\alpha(u)+\beta(u)) \gamma(v)+\gamma(u)(\tilde{\alpha}(v)+\tilde{\beta}(v))=(\alpha(v)+\beta(v)) \gamma(u)+\gamma(v)(\tilde{\alpha}(u)+\tilde{\beta}(u)) \\
\left(1-q^{2}\right)\left(\left(1-q^{2}\right)(\tilde{\alpha}(u)+\widetilde{\beta}(u)) \gamma(v)+\left(q^{u_{-}+2}-1\right)(\widetilde{\alpha}(v)+\tilde{\beta}(v)) \gamma(u)\right) \\
=\left(q^{u_{+}+2}-1\right)\left(\left(q^{u_{-}+2}-1\right) \gamma(v)(\alpha(u)+\beta(u))+\left(1-q^{2}\right) \gamma(u)(\alpha(v)+\beta(v))\right) \\
\quad-q^{2}\left(q^{u-}-1\right)\left(q^{u_{+}}-1\right)(\alpha(u)-\beta(u)) \gamma(v), \\
q^{u_{+}}\left(1-q^{2}\right)\left(q^{u_{-}}\left(1-q^{2}\right) \gamma(v)(\alpha(u)+\beta(u))+\left(q^{u_{-}+2}-1\right) \gamma(u)(\alpha(v)+\beta(v))\right) \\
=\left(q^{u_{+}+2}-1\right)\left(\left(q^{u_{-}+2}-1\right)(\tilde{\alpha}(u)+\tilde{\beta}(u)) \gamma(v)+q^{u_{-}}\left(1-q^{2}\right)(\tilde{\alpha}(v)+\tilde{\beta}(v)) \gamma(u)\right)
\end{gathered}
$$




$$
\begin{aligned}
& -q^{2}\left(q^{u-}-1\right)\left(q^{u_{+}}-1\right) \gamma(v)(\tilde{\alpha}(u)-\tilde{\beta}(u)) \\
& \left(q^{u-}-1\right)\left(\left(q^{u++2}-1\right) \gamma(u)(\alpha(v)+\beta(v))-\left(1-q^{2}\right)(\alpha(u)+\beta(u)) \gamma(v)\right) \\
& =\left(q^{u_{+}}-1\right)\left(\left(q^{u_{-}+2}-1\right)(\alpha(v)-\beta(v)) \gamma(u)+q^{u_{-}}\left(1-q^{2}\right)(\widetilde{\alpha}(u)-\widetilde{\beta}(u)) \gamma(v)\right) \text {, } \\
& \left(q^{u-}-1\right)\left(\left(q^{u_{+}+2}-1\right)(\tilde{\alpha}(v)+\widetilde{\beta}(v)) \gamma(u)-q^{u_{+}}\left(1-q^{2}\right) \gamma(v)(\tilde{\alpha}(u)+\tilde{\beta}(u))\right) \\
& =\left(q^{u_{+}}-1\right)\left(\left(q^{u_{-}+2}-1\right) \gamma(u)(\tilde{\alpha}(v)-\tilde{\beta}(v))+\left(1-q^{2}\right) \gamma(v)(\alpha(u)-\beta(u))\right), \\
& \left(q^{u_{+}}-1\right)\left(1-q^{2}\right)\left(q^{u_{-}}(\tilde{\alpha}(v)-\tilde{\beta}(v))(\alpha(u)+\beta(u))-(\tilde{\alpha}(u)-\tilde{\beta}(u))(\alpha(v)+\beta(v))\right), \\
& =\left(q^{u_{-}}-1\right)\left(1-q^{2}\right)\left(q^{u_{+}}(\dot{\alpha}(u)+\beta(u))(\alpha(v)+\beta(v))\right. \\
& -(\widetilde{\alpha}(v)-\tilde{\beta}(v))(\widetilde{\alpha}(u)-\tilde{\beta}(u)))-\left(q^{u_{-}}-1\right)\left(q^{u_{+}+2}-1\right) \gamma(u) \gamma(v), \\
& \left(q^{u_{+}}-1\right)\left(1-q^{2}\right)\left(q^{u_{-}}(\tilde{\alpha}(v)+\tilde{\beta}(v))(\alpha(u)-\beta(u))-(\tilde{\alpha}(u)+\tilde{\beta}(u))(\alpha(v)-\beta(v))\right), \\
& =\left(q^{u_{-}}-1\right)\left(1-q^{2}\right)\left(q^{u_{+}}(\alpha(u)-\beta(u))(\alpha(v)-\beta(v))\right. \\
& -(\tilde{\alpha}(v)+\widetilde{\beta}(v))(\widetilde{\alpha}(u)+\widetilde{\beta}(u)))+\left(q^{u_{-}}-1\right)\left(q^{u_{+}+2}-1\right) \gamma(u) \gamma(v), \\
& \left(q^{u_{-}}-1\right)\left((\alpha(u)-\beta(u))(\alpha(v)-\beta(v))-q^{u_{+}} F(u) F(v)\right) \\
& =\left(q^{u_{+}}-1\right)\left((\alpha(u)-\beta(u)) F(v)-q^{u_{-}}(\alpha(v)-\beta(v)) F(u)\right) \text {, } \\
& \left(q^{u_{-}}-1\right)\left((\tilde{\alpha}(u)-\tilde{\beta}(u))(\tilde{\alpha}(v)-\tilde{\beta}(v))-q^{u_{+}} F(u) F(v)\right) \\
& =\left(q^{u_{+}}-1\right)\left((\tilde{\alpha}(u)-\tilde{\beta}(u)) F(v)-q^{u_{-}}(\tilde{\alpha}(v)-\tilde{\beta}(v)) F(u)\right),
\end{aligned}
$$

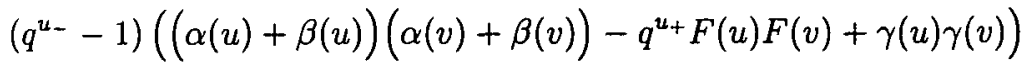

$$
\begin{aligned}
& =\left(q^{u_{+}}-1\right)\left((\alpha(u)+\beta(u)) F(v)-q^{u_{-}}(\alpha(v)+\beta(v)) F(u)\right), \\
& \left(q^{u_{-}}-1\right)\left((\tilde{\alpha}(u)+\widetilde{\beta}(u))(\tilde{\alpha}(v)+\tilde{\beta}(v))-q^{u_{+}} F(u) F(v)+\gamma(u) \gamma(v)\right) \\
& =\left(q^{u_{+}}-1\right)\left((\widetilde{\alpha}(u)+\widetilde{\beta}(u)) F(v)-q^{u_{-}}(\tilde{\alpha}(v)+\tilde{\beta}(v)) F(u)\right) \text {. }
\end{aligned}
$$

Solving these equations using some nontrivial tricks of variable separation, we have

$$
\begin{aligned}
\alpha(u)+\beta(u) & =\tilde{\alpha}(u)+\tilde{\beta}(u)=\frac{\left(q^{u+2}-q^{c}\right)\left(q^{u+2}-q^{-c}\right)}{\left(q^{u+c}-q^{2}\right)\left(q^{u-c}-q^{2}\right)} \\
\alpha(u)-\beta(u) & =\frac{q^{2 u+2}-q^{u+c+2}-q^{u-c+2}+q^{4}-q^{2}+1}{\left(q^{u+c}-q^{2}\right)\left(q^{u-c}-q^{2}\right)} \\
\tilde{\alpha}(u)-\tilde{\beta}(u) & =\frac{q^{2 u+4}-q^{2 u+2}+q^{2 u}-q^{u+c+2}-q^{u-c+2}+q^{2}}{\left(q^{u+c}-q^{2}\right)\left(q^{u-c}-q^{2}\right)} \\
\gamma(u) & =\frac{q\left(q^{2}-1\right)\left(q^{2 u}-1\right)}{\left(q^{u+c}-q^{2}\right)\left(q^{u-c}-q^{2}\right)} \\
F(u) & =\frac{\left(q^{u-c+2}-1\right)\left(q^{-u+c+2}-1\right)}{\left(q^{u+c}-q^{2}\right)\left(q^{u-c}-q^{2}\right)} .
\end{aligned}
$$


Substituting these results into (50) and (48), we may establish the non-c-number boundary $K$-matrix $K_{-}(u)$, which is a solution of the graded reflection equation (11). Using the same method, we may find the other non-c-number boundary $K$-matrix $K_{+}(u)$ by solving the dual graded reflection equation (12).

\section{REFERENCES}

[1] P.W. Anderson, 'The resonating valence bond state in $\mathrm{La}_{2} \mathrm{CuO} \mathrm{O}_{4}$ and superconductivity', Science 235 (1987), 1196-1198.

[2] A.J. Bracken, X.-Y. Ge, Y.-Z. Zhang and H.-Q. Zhou, 'Integrable open-boundary conditions for the $q$-deformed supersymmetric $U$ model of strongly correlated electrons', Nuclear Phys. B 516 (1998), 588-602.

[3] F.H.L. Essler and V.E. Korepin, 'Higher conservation laws and algebraic Bethe ansatz for the supersymmetric $t-J$ model', Phys. Rev. B 46 (1992), 9147-9162.

[4] F.H.L. Essler and V.E. Korepin, 'Spectrum of low-lying excitations in a supersymmetric extended Hubbard model', Internat. J. Modern. Phys. B 8 (1994), 3243-3279.

[5] F.H.L. Essler, V.E. Korepin and K. Schoutens, 'New exactly solvable model of strongly correlated electrons motivated by high $T_{c}$ superconductivity', Phys. Rev. Lett. 68 (1992), 2960-2963.

[6] F.H.L. Essler, V.E. Korepin and K. Schoutens, 'Electronic model for superconductivity', Phys. Rev. Lett. 70 (1993), 73-76.

[7] F.H.L. Essler, V.E. Korepin and K. Schoutens, 'Exactly solution of an electronic model of superconductivity', Internat. J. Modem. Phys. A 8 (1994), 3205-3242.

[8] H. Fan, M. Wadati and R.-H. Yue, 'Boundary impurities in the generalized supersymmetric $t-J$ model', J. Phys. A 33 (2000), 6187-6202.

[9] H. Frahm and N.A. Slavnov, 'New solution of the reflection equation and the projecting method', J. Phys. A 32 (1999), 1547-1555.

[10] X.-Y. Ge, 'Integrable open-boundary conditions for the $q$-deformed extended Hubbard model', Modern. Phys. Lett. B 13 (1999), 499-507.

[11] X.-Y. Ge, M.D. Gould, J. Links and H.-Q. Zhou, 'Integrable Kondo impurity in one-dimensional $q$-deformed $t-J$ models', J. Phys. $A$ (to appear).

[12] Z.-N. Hu, F.-C. Pu and Y. Wang, 'Integrability of the $t-J$ model with impurities', $J$. Phys. A 31 (1998), 5241-5262.

[13] P. Schlottmann and A.A. Zvyagin, 'Kondo impurity band in a one-dimensional correlated electron lattice, Phys. Rev. B 56 (1997), 13989-13998.

[14] K. Schoutens, 'Complete solution of a supersymmetric extended Hubbard model', Nuclear Phys. B 413 (1994), 675-688.

[15] B.S. Shastry, 'Exact integrability of the one-dimensional Hubbard model', Phys. Rev. Lett. 56 (1986), 2453-2455.

[16] B.S. Shastry, 'Decorated star-triangle relations and exact integrability of the one-dimensional Hubbard model', J. Statist. Phys. 50 (1988), 57-79.

[17] E.K. Sklyanin, 'Boundary conditions for integrable quantum systems', J. Phys. A 21 (1988), 2375-2389.

[18] E.K. Sklyanin, L.A. Takhtajan and L.D. Faddeev, 'Quantum inverse problem method I', Theoret. and Math. Phys. 40 (1980), 688-706. 
[19] Y. Wang, J.-H. Dai, Z.-N. Hu and F.-C. Pu, 'Exact results for a Kondo problem in one dimensional $t-J$ model', Phys. Rev, Lett. 79 (1997), 1901-1904.

[20] F.C. Zhang and T.M. Rice, 'Effective Hamiltonian for the superconducting $C u$ oxides', Phys. Rev. B 37 (1988), 3759-3761.

[21] H.-Q. Zhou, X.-Y. Ge and M.D. Gould, 'Integrable Kondo impurities in the one-dimensional supersymmetric extened Hubbard model', J. Phys. A 32 (1999), 5383-5388.

[22] H.-Q. Zhou, X.-Y. Ge, J. Links and M.D. Gould, 'Graded reflection equation algebras and integrable Kondo impurities in the one-dimensional $t-J$ model', Nuclear. Phys. $B$ 546 (1999), 779-799.

[23] H.-Q. Zhou, X.-Y. Ge, J. Links and M.D. Gould, 'Integrable Kondo impurities in the one-dimensional extended Hubbard models', Phys. Rev. B 62 (2000), 4906-4921.

[24] H.-Q. Zhou and M.D. Gould, 'Algebraic Bethe ansatz for integrable Kondo impurities in the one-dimensional supersymmetric $t-J$ model', Phys. Lett. A 251 (1999), 279-285.

[25] A.A. Zvyagin and P. Schlottmann, 'Exact solution for a one-dimensional multichannel model of correlated electrons with an Anderson-like impurity', J. Phys. A 31 (1998), 1981-1987.

Centre for Mathematical Physics

The University of Queensland

Brisbane, Qld 4072

Australia

e-mail: $\quad x g @ m a t h s . u q . e d u . a u$ 